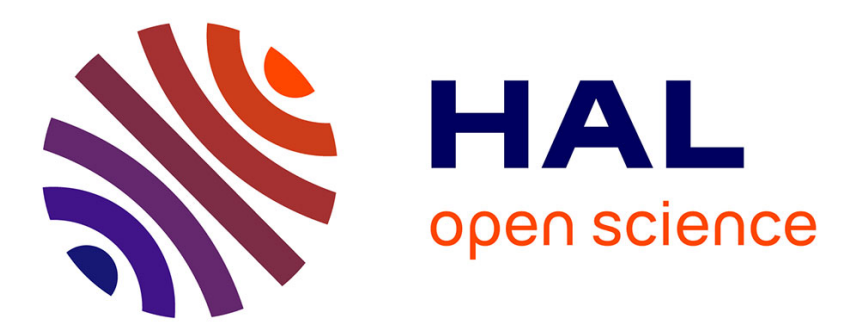

\title{
Monte-Carlo and multifluid modelling of the circumnuclear dust coma II. Aspherical-homogeneous, and spherical-inhomogeneous nuclei
}

\author{
V.V. Zakharov, A.V. Rodionov, G.A. Lukianov, Jean-François Crifo
}

\section{- To cite this version:}

V.V. Zakharov, A.V. Rodionov, G.A. Lukianov, Jean-François Crifo. Monte-Carlo and multifluid modelling of the circumnuclear dust coma II. Aspherical-homogeneous, and spherical-inhomogeneous nuclei. Icarus, 2009, 201 (1), pp.358-380. 10.1016/j.icarus.2008.12.022 . hal-00524851

\section{HAL Id: hal-00524851 \\ https://hal.science/hal-00524851}

Submitted on 9 Oct 2010

HAL is a multi-disciplinary open access archive for the deposit and dissemination of scientific research documents, whether they are published or not. The documents may come from teaching and research institutions in France or abroad, or from public or private research centers.
L'archive ouverte pluridisciplinaire HAL, est destinée au dépôt et à la diffusion de documents scientifiques de niveau recherche, publiés ou non, émanant des établissements d'enseignement et de recherche français ou étrangers, des laboratoires publics ou privés. 


\section{Accepted Manuscript}

Monte-Carlo and multifluid modelling of the circumnuclear dust coma II. Aspherical-homogeneous, and spherical-inhomogeneous nuclei

V.V. Zakharov, A.V. Rodionov, G.A. Lukianov, J.F. Crifo

PII: $\quad$ S0019-1035(08)00450-8

DOI: $\quad$ 10.1016/j.icarus.2008.12.022

Reference: $\quad$ YICAR 8860

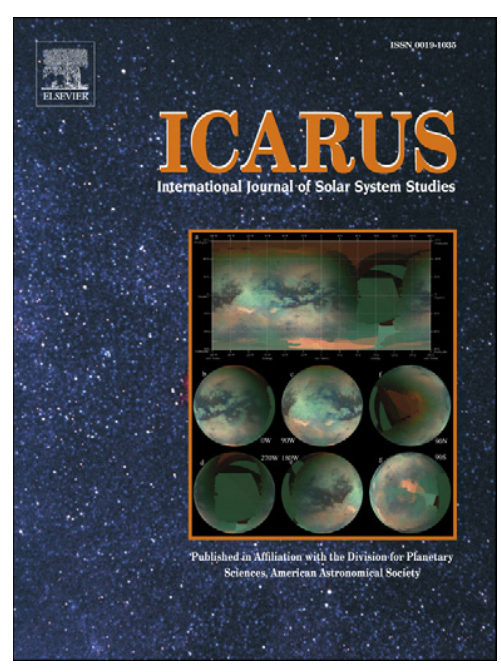

To appear in: Icarus

Received date: 7 May 2008

Revised date: 19 November 2008

Accepted date: 12 December 2008

Please cite this article as: V.V. Zakharov, A.V. Rodionov, G.A. Lukianov, J.F. Crifo, Monte-Carlo and multifluid modelling of the circumnuclear dust coma II. Aspherical-homogeneous, and spherical-inhomogeneous nuclei, Icarus (2009), doi: 10.1016/j.icarus.2008.12.022

This is a PDF file of an unedited manuscript that has been accepted for publication. As a service to our customers we are providing this early version of the manuscript. The manuscript will undergo copyediting, typesetting, and review of the resulting proof before it is published in its final form. Please note that during the production process errors may be discovered which could affect the content, and all legal disclaimers that apply to the journal pertain. 


\section{Monte-Carlo and Multifluid Modelling of the Circumnuclear}

Dust Coma II. Aspherical-Homogeneous, and

Spherical-Inhomogeneous Nuclei

V.V. Zakharov ${ }^{* \ddagger}$, A.V. Rodionov ${ }^{\dagger}$, G.A. Lukianov ${ }^{\ddagger \S}$, J.F. Crifo
Submitted to ICARUS : May 7, 2008

Revised: November 19, 2008

Approved: December 16, 2008

Manuscript NR: I10485

Number of double-space manuscript pages (including Figs and tables): 59

Number of Figures: 14

Number of Tables: 4

\section{Running Title: Multifluid and Monte-Carlo Dust Coma Simulations II}

*LESIA, Observatoire de Paris, F92195 Meudon Cedex, France zvv1661@yandex.ru

${ }^{\dagger}$ Central Research Institute on Machine Building (TsNIIMASH), Pionyerskaya st., 4, Korolev, Moscow Region 141070, Russia

${ }^{\ddagger}$ Center for Advanced Studies, St.-Petersburg State Technical University, Polytechnicheskaya str., 29, 195251 St.-Petersburg, Russia

${ }^{\S}$ We are sorry to announce that Professor Lukianov deceased suddenly on June 5, 2007.

`Service d’Aéronomie du CNRS, BP 3, F 91371 Verrières le Buisson Cedex, France. crifo@aerov.jussieu.fr 
Send correspondence to:

Jean-François CRIFO

CNRS, Service d'Aéronomie, BP 3, F 91371 Verrières Cedex, France

Telephone: $(+33)$ 1.64.47.42.72

18

e-mail: $\quad$ crifo@aerov.jussieu.fr

\section{Running Title:}

Multifluid and Monte-Carlo Dust Coma Simulations - II 


\section{Abstract}

We use our newly developed Dust Monte-Carlo (DMC) simulation technique (Crifo et al., 2005a) to study the dynamics of dust grains in the vicinity of some of the benchmark aspherical, homogeneous cometary nuclei and of the benchmark spherical, inhomogeneous nuclei studied by us precedingly. We use the interim unrealistic simplifying assumptions of grain sphericity, negligible nucleus rotation rate, and negligible tidal force, but take accurately into account the nucleus gravitational force, gas coma aerodynamic force, and solar radiation pressure force, and consider the full mass range of ejectable spherical grains. The resulting complicated grain motions are described in detail, as well as the resulting complicated and often counter-intuitive dust coma structure. The results are used to answer several important questions: (1) When computing coma dust distributions, (a) is it acceptable to take into consideration only one or two of the above mentioned forces (as currently done)? (b) to which accuracy must these forces be known, in particular is it acceptable to represent the gravity of an aspherical nucleus by a spherically symmetric gravity? (c) how do the more efficient but less general Dust Multi-Fluid (DMF) computations compare with the DMC results? (2) Are there simple structural relationships between the dust coma of a nucleus at small heliocentric distance $r_{h}$, and that of the same nucleus at large $r_{h}$ ? (3) Are there similarities between the gas coma structures and the associated dust coma structures? (4) Are there dust coma signatures revealing non-ambiguously a spherical nucleus inhomogeneity or an homogeneous nucleus asphericity? (5) What are the implications of the apparently quite general process of grain fall-backs for the evolution of the nucleus surface, and for the survival of a landed probe?

\section{Introduction}

This paper is the second of a series of advanced theoretical studies of the dust dynamics and distribution in the immediate vicinity of an active cometary nucleus. By "advanced" modelling, we mean modellling capable of taking into account all observed quirks of cometary nuclei and grains, as revealed for the first time by the 1986 flyby observations of comet 
Halley, and, more recently, by the remarkable flybys of comets P/Borrelly, P/Tempel I, and $\mathrm{P} /$ Wild 2, and expected from the forthcoming rendez-vous mission Rosetta. Such an objective can only be met by a carefully planned effort involving successive steps of increased sophistication. This is because the results from a sophisticated code involving numerous input parameters can only be understood if the effect of each parameter is already known. The optimization of the code, also, requires omission of those of these quirks which have been demonstrated to be negligible. Thus, our effort is somewhat similar to a succession of simulated laboratory experiments in which the samples studied are designed in such a way that different effects are studied separately. Such a method is also ideally suitable for comparing various mathematical methods of modelling of the dust coma, hence we also give a large importance to this objective.

Our first work (Crifo et al., 2005a, hereafter referred to as "paper D-I", Lukyanov et al., 2006) described our general objectives and our approach. The simplest possible benchmark nuclei were postulated: several non-rotating spheres assumed to be a homogeneous mixture of water ice and spherical, homogeneous mineralic grains. The gas comae formed by solardriven ice sublimation from these nuclei had been previously computed in Crifo et al. (2002) (hereafter paper G-I). The grains were assumed to be isothermal - so that they are not set in rotation - and free from volatile material (i.e. non-sublimating and non-condensing). The solar tidal force was not taken into account, nor mutual dust collisions. On the other hand, it was the first time that three of the many forces acting on the grains were taken into account simultaneously: the aerodynamic drag, the radiation pressure, and the nuclear gravity. For the first time also, we used two independent and complementary methods - the so-called "dust multifluid" (DMF) method (see Rodionov et al., 2002 and references therein) - and the so-called Dust Monte-Carlo method (DMC) to treat the same problems. This Monte-Carlo approach bears more similarities to the Monte-Carlo modelling of planetary atmospheres (e.g., Hodges, 1994) than to the so called "Direct Monte Carlo Simulation" (DSMC) of rarefied gas flows, because of the dominant role of the external force(s). For that reason we prefer, from now on, to call it "DMC" instead of "DSMC" used in paper 
D-I.

The present paper still retains the preceding simplistic assumptions, except the following: we consider here the nucleus to be either one of the "homogeneous, aspherical" nuclei described in Crifo et al. (2003) (hereafter paper G-II), or one of the "spherical, inhomogeneous" nuclei described in Zakharov et al. (2008) (hereafter paper G-III). These nuclei are axially symmetric, and the sun is assumed placed on their symmetry axis, illuminating the "interesting side" of the nucleus, i.e. where the inhomogeneous or aspherical part lies.

It is important to remind here very precisely what we mean by "inhomogeneity". As indicated in G-III, all nuclei we consider, being ice-dust mixtures, are inhomogeneous on a microscopic scale (comparable to the dust grain radii and intergrain spacing ). To this miscroscopic inhomogeneity is associated the so-called icy area fraction $f$, which is the fraction of a small surface element consisting of exposed ice, the rest being non-icy dust (see Crifo, 1997 and Rodionov et al., 2002). It is related to the total dust-to-gas mass ratio $\Re$ in the ice at this point by the relation (Crifo, 1997):

$$
f=1 /\left[1+\left(\rho_{I} / \rho_{d}\right) \Re\right]
$$

where $\rho_{I}$ and $\rho_{d}$ are, respectively, the ice and the dust specific mass. Equation 1 holds because we assume that all dust grains have the same $\rho_{d}$, irrespective of their size. We will furthermore assume (for simplicity) that $\rho_{d}=\rho_{I}=1000 \mathrm{~kg} \mathrm{~m}^{-3}$ everywhere, whereby the nucleus specific mass is $\rho_{n}=1000 \mathrm{~kg} \mathrm{~m}^{-3}$. If $f$ is the same everywhere on the nucleus surface, we call the nucleus "homogeneous" (on a macroscopic scale much greater than the microscopic one), whereas, if $f$ varies from point to point on such a macroscopic scale, we call the nucleus "inhomogeneous" (i.e., on a macroscopic scale).

The gas comae around the nuclei considered here have been extensively described in papers G-II and G-III. They are characterized by a wealth of structures. One of the goals of the present work is to compute the corresponding near-nucleus dust coma structures.

To keep the size of this paper reasonable, and also in view of the interim nature of the assumptions on which it is based, we neither describe, nor even show the night side part 
of the present solutions. This is not to suggest that the night side grain dynamics is of no interest. Even though the night side surface of our model nuclei is practically hemispherical and homogeneous, as in paper D-I, it is true that the aerodynamic force field experienced there by particles arriving from the dayside may differ significantly from what they would experience if the nucleus was strictly spherical. This is because, as shown in paper D-I, section 7.4, many grain trajectories present in the night side coma are extremely sensitive to even very small changes in the initial conditions and/or applied forces experienced by the grains in the day side. Strong differences in applied forces experienced on the day side exist, resulting from (1) differences in the dayside gas flow, and (2) differences in the gravitational field (as we will show). However, the results of paper I show that grains arriving on the dayside from the nightside coma do not influence dramatically the dayside grain distribution hence it is permitted to treat the dayside separately, and to study the night side in a future work.

\section{Physical model}

We consider the four axially symmetric dusty ice nuclei shown on Figure 1, selected among those described in papers G-II and G-III: two aspherical, homogeneous surfaces (labelled with the prefix "top" and "app" as in paper G-II), and two spherical, inhomogeneous surfaces (labelled with the suffix $z 1$ and $z 2$ as in paper G-III). For each of these nuclei, we assume two very different levels of gas production: a very high one, labelled \#207D as in paper G-III, and a rather low one, labelled \#205 as in papers G-I, G-II, G-III. In passing, let us give here a precision that was not given in our previous papers: we call $Q$ the net upward flux at the top of the sublimation boundary layer. It implies that we do not subtract from it any downward flux that could exist near to condensing parts of the nucleus surface. Thus, strictly speaking, our $Q$ may be slightly in excess of what is usually called $Q$, i.e., the total flux of vapor escaping to infinity.

Table 1 summarizes the parameters of the resulting eight different cases considered; the definition of these parameters is given in the following. Let us remember that the sun 

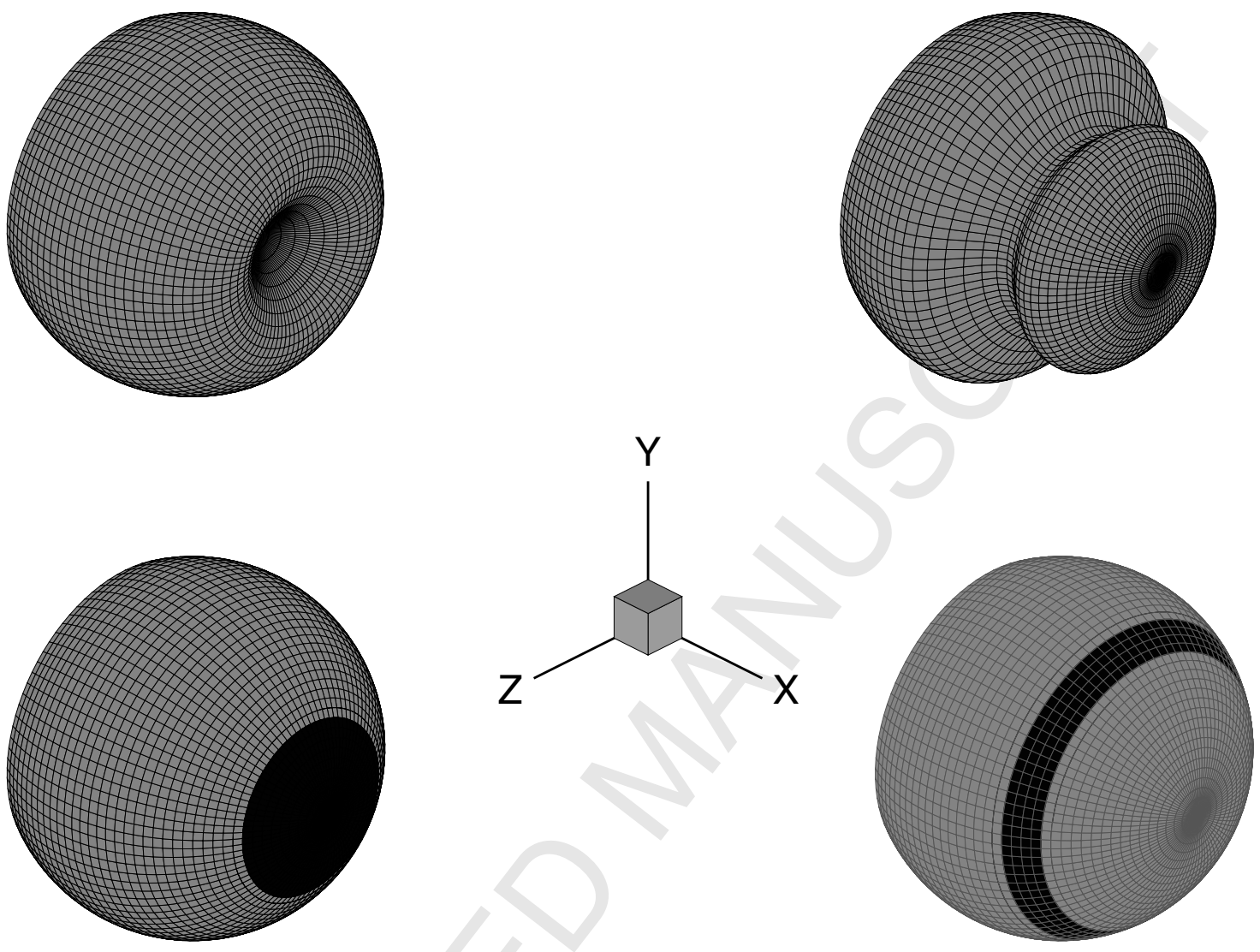

Figure 1: Model benchmark nuclei used in this study. Top panels: Homogeneous nuclei. Top Left: apple-shaped nucleus with shape parameter value $P=0.6$. Top Right: top-shaped nucleus with shape parameter value $P=-0.21$. Bottom panels: Spherical, inhomogeneous nuclei. The region of low icy area fraction $f$ is in dark. Bottom Left: nuclei of kind $z 1$, which have a weakly active cap; Bottom Right nuclei of kind $z 2$, which have a weakly active ring. For the present computations, the sun is always assumed to be in the $+\mathrm{X}$ direction. 
direction is the axis of symmetry OX.

\subsection{Aspherical, homogeneous nuclei}

We consider here the two aspherical, homogeneous nuclei called "apple" and "top", shown in Figure 1, and defined by Eqs (1) and (2) of paper G-II, where the characteristic dimension $R$, which defines the size, and the dimensionless parameter $P$, which defines the shape, are given in Table 1. The "apple" nuclei have a large funnel-like subsolar cavity, and the "top" nuclei have a mid-latitude valley encircling the symmetry axis. These nuclei are assumed to be homogeneous, i.e., to have a uniform icy area fraction $f \leq 1$.

The volumes $\mathcal{V}_{n}$ of the "top" and "apple" defined by the same $R$ value differ from one another, and from that of a sphere with radius $R$ (see Table 1). Hence their masses $M_{n}=\rho_{n} \mathcal{V}_{n}$ differ from one another, and from the reference mass $M_{n}^{\star}=(4 \pi / 3) \rho_{n} R^{3}$. Let us also note that the center-of-mass (c.o.m.) of these nuclei does not coincide with the origin of the coordinates: the c.o.m. abscissa $X^{C O M}$ is given in Table 1. Most importantly, the "top" and "apple" nuclei give birth to two genuine non-radial and position-dependent gravity fields. This point was immaterial when discussing the gas coma, which is insensitive to the nucleus gravity, but is consequential when discussing the dust coma, since dust is sensitive, in general, to the gravity field. As we will see, the gravitational field asphericity has an effect, not only on the grains trajectories, but, as well, on the value of the maximum liftable mass at each point.

In all previous works where dust ejected from an aspherical nucleus was considered (DMF computations reviewed in Crifo et al.,1999, Crifo et al., 2005b, and Crifo, 2006) an origin-centered spherically symmetric gravity field with mass $M_{n}^{\star}$ was used ${ }^{1}$. This raises some concern, since the best-fit $1 / r^{2}$ approximation to the gravitational field of an aspherical object must (1) be centered at the c.o.m. - different from the origin - and (2) correspond to the mass $M_{n}-$ not $M_{n}^{\star}$. Furthermore, the best-fit $M_{n} / r^{2}$ approximation holds only outside

\footnotetext{
${ }^{1}$ It is of course possible to use the true gravity in DMF computations, but this has not been done up to now, because the importance of using the true gravity has been overlooked.
} 


\begin{tabular}{|c|c|c|c|c|c|c|c|c|}
\hline ACRONYM & app207D & top207D & 207D_z1 & 207D_z2 & app205 & top205 & 205_z1 & 205_z2 \\
\hline$r_{h}(\mathrm{AU})$ & 1.0 & 1.0 & 1.0 & 1.0 & 5.79 & 5.79 & 5.79 & 5.79 \\
\hline$R(\mathrm{~km})$ & 5.0 & 5.0 & 5.0 & 5.0 & 6.95 & 6.95 & 6.95 & 6.95 \\
\hline$P$ & 0.6 & -0.21 & 0 & 0 & 0.6 & -0.21 & 0 & 0 \\
\hline$\kappa$ & 0.01 & 0.01 & 0.01 & 0.01 & 0.6 & 0.6 & 0.6 & 0.6 \\
\hline$z^{\max }\left({ }^{0}\right)$ & - & - & 30 & 65 & - & & & 30 \\
\hline$z^{\min }\left({ }^{0}\right)$ & - & - & - & 50 & - & & & 15 \\
\hline$f_{\max }$ & 0.3 & 0.3 & 0.3 & 0.3 & 1 & & 1 & 1 \\
\hline$f_{\min }$ & 0.3 & 0.3 & 0.03 & 0.03 & 1 & & 0.1 & 0.1 \\
\hline$r_{\max }(\mathrm{km})$ & 50 & 50 & 25 & 25 & 70 & 70 & 70 & 70 \\
\hline $\mathcal{V}_{n}\left(\mathrm{~km}^{3}\right)$ & 497 & 442 & 523 & 523 & 1336 & 1186 & 1406 & 1406 \\
\hline$X^{C O M}(\mathrm{~km})$ & -0.21 & -0.35 & 0 & 0 & -0.29 & -0.48 & 0 & 0 \\
\hline$M_{n}(\mathrm{~kg})$ & $5.0+14$ & $4.4+14$ & $5.2+14$ & $5.2+14$ & $1.3+15$ & $1.2+15$ & $1.4+15$ & $1.4+15$ \\
\hline$M_{n}^{\star}(\mathrm{kg})$ & $5.2+14$ & $5.2+14$ & $5.2+14$ & $5.2+14$ & $1.4+15$ & $1.4+15$ & $1.4+15$ & $1.4+15$ \\
\hline$Q($ molec $/ \mathrm{s})$ & $3.3+29$ & $3.3+29$ & $5+29$ & $2.6+29$ & $3.3+26$ & $3.3+26$ & $1.1+26$ & $1.85+26^{\dagger}$ \\
\hline$a_{d}^{M}(0)(\mathrm{m})$ & $1.0-1$ & $7.6-2$ & $6.9-3$ & $6.9-2$ & $1.1-4$ & $9.3-5$ & $8.3-6$ & $8.4-5$ \\
\hline$a_{d}^{M M}(\mathrm{~m})$ & $"$ & & $5.8-2$ & $"$ & $"$ & $"$ & $2.2-5$ & $"$ \\
\hline
\end{tabular}

Table 1: Parameters of the nuclei discussed in this study (above the horizontal line) and a few computed properties (below the line). The acronym prefix "top" refers to top-shaped homogeneous nuclei $(P<0)$, "app" to apple-shaped homogeneous nuclei $(P>0)$; the acronyms suffixes "z1" and "z2" refer to the spherical $(P=0)$ inhomogeneous nuclei with, respectively, a low-activity cap and a low-activity ring. The expression $a \pm b$ is used for $a \times 10^{ \pm b}$. The distance $r_{\max }$ is that of the outer boundary of the computational domain. The value marked with a $\dagger$ was misprinted in paper G-III. 
the circumscribed sphere to the central object (Garmier and Barriot, 2001); hence, inside cavities, there might not exist any good $1 / r^{2}$ approximation to the field.

Figure 2 shows the true gravity isocontours and the true gravitational field lines of the "top" and "apple" nuclei, as well as those of origin-centered $M_{n}^{\star} / r^{2}$ field used in the past. The direction of the gravity at the surface is indicated by lines drawn inside the nucleus. One sees that neither the "top" surface field lines, nor the "apple" surface field lines converge to a point: this evidences the non-existence of an accurate spherical approximation (for the surface and near-surface field). Of interest also is the fact that the gravity magnitude has a local maximum inside the "apple" cavity, but no such local maximum inside the "top" cavity. Finally, one sees that the gravity has a substantial component parallel to the flanks of the cavities, suggesting the possibility of "downslope" grain rolling.

\subsection{Inhomogeneous, spherical nuclei}

The two spherical, inhomogeneous nuclei are the "weakly active cap" and "weakly active ring" spherical nuclei (with radius $R$ ) described in paper G-III and represented on Fig. 1.

The "weakly active cap" nuclei, labelled by the acronym " $z 1$ ", have a surface icy fraction $f=f_{\text {max }}$, save within a spherical cap of opening $z^{\max }$ centered onto the solar direction, where $f$ assumes a reduced value $f_{\min }$.

As indicated in paper G-III, this problem bears similarities with the problem treated by Knollenberg (1994), and Keller et al. (1994) - that is, a circular inactive disk of 0.3 $\mathrm{km}$ radius inside a concentric active area of about $1 \mathrm{~km}$ radius. It has also a relationship to the presently discussed "apple" nucleus, which also has a reduced surface gas flux in its subsolar cavity due to the cavity wall inclination to the solar direction.

The "weakly active circular ring" nuclei, labelled by the acronym " $z 2$ ", have a surface icy fraction $f=f_{\max }$, save within a ring defined by $z^{\min } \leq z_{\odot} \leq z^{\max }\left(z_{\odot}\right.$ being the solar zenith angle) where $f$ assumes a reduced value $f_{\min }$. Notice that the position of the ring is not the same for all nuclei (see Table 1). For axial illumination, these nuclei bear similarities with the "top" shaped nuclei: in both cases, there is a ring of reduced gas production, due 

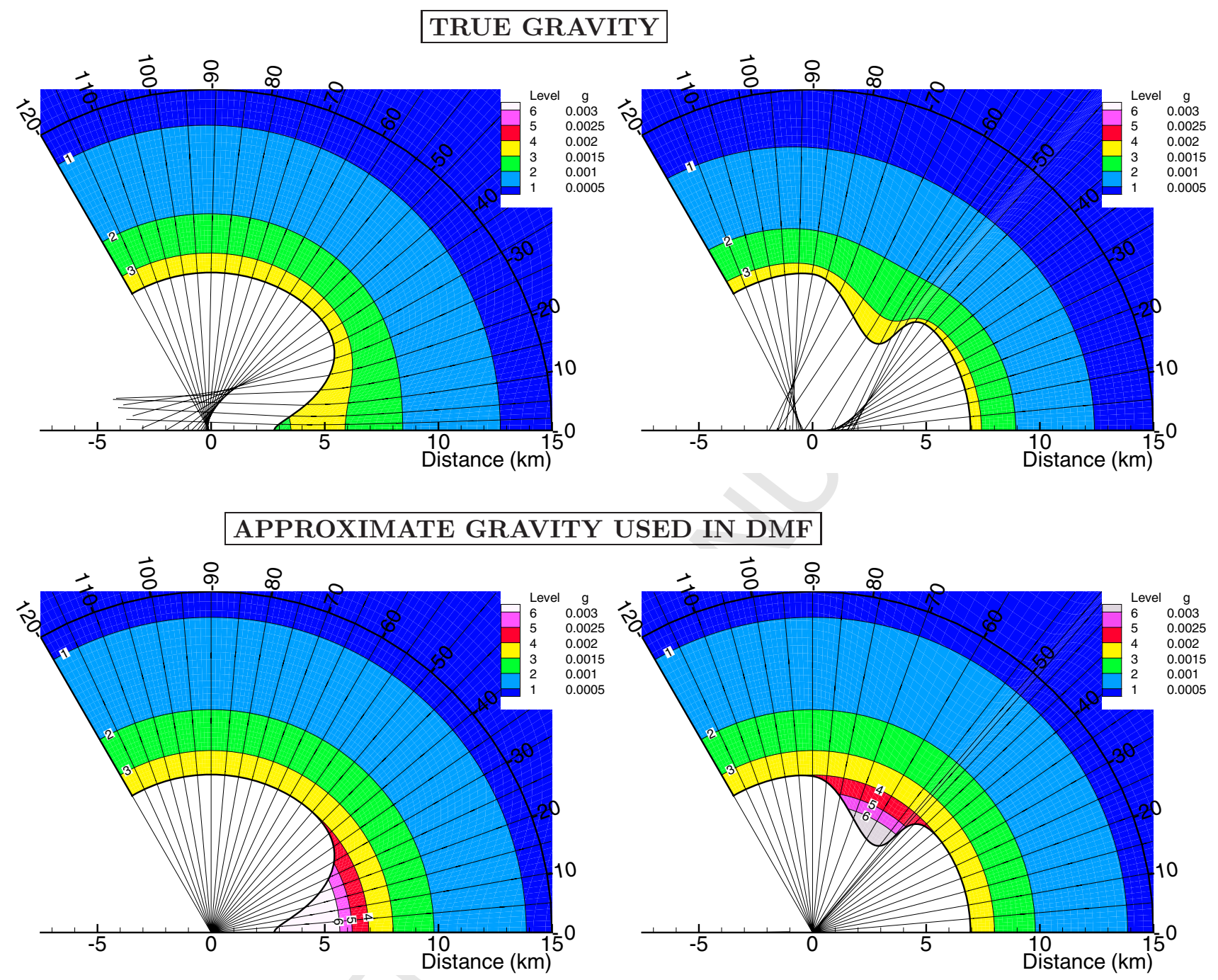

Figure 2: Gravitational field models for the aspherical nuclei \#205. Top: true (aspherical) field (in $\mathrm{m} / \mathrm{s}^{2}$ ). Bottom: origin-centered spherical field for the mass $M_{n}^{\star}$. The radial lines outside of the nucleus are the gravitational field lines; inside the nucleus, they indicate the direction of the surface field (not the internal field lines!). 
to a low $f$ or to a shadowed valley. Paper G-III revealed indeed some similarity in the gas flow patterns; we will see what happens for the dust coma.

\subsection{Dust model}

As in paper D-I, (1) spherical grains with radius $a_{d}^{s}$ and mass $m_{d}^{s}$ are considered to be submitted to the aerodynamic force, to the nucleus gravity, and to the solar radiation pressure; (2) the aerodynamic force is computed assuming a constant (size and heliocentric distance dependent) grain temperature $T_{d}$ given in Table 2, equal to the free-space temperature of impure amorphous olivine grains (i.e., having their optical absorption increased at visible wavelengths, see Crifo, 1988); (3) the radiation pressure efficiency $Q_{p r}$, also given in table 2 , is computed with the same assumption.

One important difference with paper D-I appears when dealing with the gravity. While the gravity of the spherical nuclei \#z1 and \#z2 is trivially evident, for the "apple" and "top" nuclei, the gravitational field is not spherically symmetric.

Another important difference with paper D-I appears when dealing with the inhomogeneous nuclei \#z1 and \#z2. In this case, since $f$ varies with position, we must also postulate a variation of $\Re$ with position, hence a variation of the dust mass spectrum. This is done as follows. We consider that, everywhere are present inside the ice seven different kinds of grains with the possible radii $a_{d}^{s}(1 \leq s \leq 7)$ and with the relative dust-to-ice mass ratios $\chi^{s}$. The values of $a_{d}^{s}$ and $\chi^{s}$ are given in Table 2. As in our previous works, (see Crifo and Rodionov 1997a) the latter are adopted from the in-situ measured P/Halley non-power-law dust spectrum of McDonnell et al. (1991). For $1 \leq s \leq 6$, the values of $a_{d}^{s}$ are the same in all nuclei considered, but for $s=7 a_{d}^{7}$ depends upon the kind of nucleus considered.

For the weakly active nuclei $\# 205$, unable to eject cm-size grains, we assume $a_{d}^{7}=91 \mu \mathrm{m}$ (the so-called size \#24 defined in Crifo and Rodionov, 1997a). The resulting total dust-toice mass ratio is $\Re=\sum_{s=1}^{7} \chi^{s}=0.028$. Eq. 1 shows that this corresponds to $f=0.98$ which we take as adequate at points where our model postulates $f=1$. At points where our model assumes $f=0.1$, we assume that, to the grains of Table 2, are added other 


\begin{tabular}{|l|cccccc|ccccc|}
\hline$s$ & 1 & 2 & 3 & 4 & 5 & 6 & \multicolumn{5}{|c|}{7} \\
\hline size index & 05 & 12 & 18 & 19 & 20 & 23 & 24 & 35 & 40 & 41 & 42 \\
\hline$a_{d}^{s}(\mathrm{~m})$ & $6.2-8$ & $9.1-7$ & $9.1-6$ & $1.34-5$ & $1.96-5$ & $6.2-5$ & $9.1-5$ & $6 .-3$ & $4.2-2$ & $6.2-2$ & $9.1-2$ \\
$m_{d}^{s}(\mathrm{~kg})$ & $1 .-18$ & $3.2-15$ & $3.2-12$ & $1.0-11$ & $3.2-11$ & $1.0-10$ & $3.2-9$ & $1 .-3$ & $3.2-1$ & 1.0 & 3.2 \\
$Q_{p r}$ & 0.13 & 1.25 & 1.05 & 1.02 & 1.00 & 0.98 & 1.0 & 1.0 & 1.0 & 1.0 & 1.0 \\
$T_{d}^{205}$ & 166. & 162. & 109. & 109. & 112. & 114. & - & - & - & - & - \\
$T_{d}^{207 D}$ & 334. & 326. & 250. & - & - & - & 271. & 271. & 271. & 271. & 271. \\
$\chi^{s}$ & $2.62-5$ & $6.11-3$ & $5.0-3$ & $3.47-3$ & $3.47-3$ & $3.39-3$ & $6.2-3$ & $3.0-1$ & $5.3-1$ & $6.0-1$ & $6.6-1$ \\
\hline
\end{tabular}

Table 2: Spherical impure amorphous olivine grains considered in this study. The "size index" refers to the position of the grain size within the Halley-like spectrum defined in Crifo and Rodionov (1997a). The meaning of the symbols is given in the text. The expression $a \pm b$ is used for $a \times 10^{ \pm b}$.

grains with mass much greater than the maximum ejectable mass, and in abundance such that they provide the desired value of $f$. Since these grains are not ejected, they need not be better specified.

For the highly active nuclei \#207D, we choose in alternance $a_{d}^{7}=0.6,4.2,6.2$ or $9.1 \mathrm{~cm}$, in order to explore the dynamics of grains in this size range (these grains are the so-called \#35, \#40, \#41 and \#42 grains defined in Crifo and Rodionov, 1997a). The resulting total dust-to-ice mass ratio $\Re=\sum_{s=1}^{7} \chi^{s}$ varies from $\simeq 0.3$ to $\simeq 0.7$. Eq. 1 shows that this corresponds to $f$ values from $\simeq 0.77$ to $\simeq 0.59$. Thus, for these nuclei, to obtain the values 0.3 and 0.03 postulated for $f$, we must assume the presence (in adhoc amounts) of large non ejectable pebbles, not only in the low activity areas but, as well, in the (relatively) high activity areas.

It would surely be permitted to make differing assumptions, for instance, that the size distribution differs completely between the highly active and the weakly active areas, or that the specific mass differs. We will not play with such possibilities here, for the sake 
of brevity. However, the reader concerned about the interpretation of dust coma images should be careful not to forget that the word "inhomogeneity" may designate quite different physical structures.

If the dust size distribution extends to masses large enough that they cannot be ejected, then it is evident that the nucleus surface composition becomes time-dependent (by selective depletion of the ejectable grains). This is also the case if dust detached from the surface fall-backs onto it, as in the "volcanoes" described for the first time in paper D-I, and further described below. We do not try to model such a time-dependence: it must be understood that our solutions hold for "some time" during which the composition of the surface has not been changed significantly.

\subsection{Gas and dust production}

The net $\mathrm{H}_{2} \mathrm{O}$ flux (molecule $/ \mathrm{cm}^{2} \mathrm{~s}$ ) at each point with solar zenith angle $z_{\odot}$ is set equal to $f Z\left[T_{I}\left(z_{\odot}\right), M_{0}\left(z_{\odot}\right)\right]$, where $Z\left(T_{I}, M_{0}\right)$ is the pure ice net sublimation flux at ice temperature $T_{I}$ and initial flow Mach number $M_{0}$; these initial flow parameters are computed by coupling the classical surface energy budget equation at each point to the gas flow governing equations, and solving the whole set simultaneously. For the chosen on-axis solar illumination, the resulting total $\mathrm{H}_{2} \mathrm{O}$ production rate $Q$ depends upon the parameters defining the nucleus size, shape, and inhomogeneity, and upon its heliocentric distance $r_{h}$. We consider the two typical combinations indicated in Table 1, selected among the cases previously considered in papers G-I, G-II and G-III: (1) a large size, highly active nucleus (label \#207D); (2) a large size, weakly active nucleus (label \#205). The set \#207D is more or less suitable for a comet like comet Halley at the Earth's orbit. The set \#205 has cannot have any observed counterpart, since its production rate is too low for detection near Jupiter's orbit. It represents an intermediate size, modestly active icy body at that distance. Most likely, the gas production of comets at that distance is dominated by $C O$ or other volatile molecules, but for the present study changing the molecular species would be an unnecessary complication. 
The parameter $\kappa$ is introduced to simulate a heat flux from the nucleus interior at points in shadow (to prevent the surface temperature from dropping to zero), so that, at such points, a finite (and small) sublimating gas flux exists, which depends upon $f, \kappa$, and $r_{h}$.

The inhomogeneous nuclei \#207D were precedingly discussed in paper G-III. As to the aspherical nuclei \#207D, they were not dicussed. Instead, identical aspherical nuclei having a ten times smaller $f$ value were considered in paper G-II (nuclei \#207d). These two families of nuclei differ only by their $f$ value, hence by their total gas production rates, which are nearly exactly proportional to $f$. Their dayside near-nucleus gas comae are in strict inviscid flow regime, thus are "similar" in the gasdynamical sense: at any point, the gas temperature and velocity are the same, and the gas density scales according to the ratio of the $f$ values. The reader can therefore easily picture the dayside gas comae of the aspherical nuclei \#207D by referring to the Figures of paper G-II for \#207d, and scaling-up the gas density by a factor 10 .

The surface flux $Z_{d}^{s}\left(z_{\odot}\right)$ (grain $/ \mathrm{cm}^{2} \mathrm{~s}$ ) of grains of size $s$ at any point $z_{\odot}$ of the surface is by definition of $\chi^{s}$ equal to:

$$
Z_{d}^{s}\left(z_{\odot}\right)=\left(m_{g} / m_{d}^{s}\right) \chi^{s} f Z\left[\left(T_{I}\left(z_{\odot}\right), M_{0}\left(z_{\odot}\right)\right]\right)
$$

provided that the normal component of the sum of the applied forces is outwards at the considered point, and this flux is zero otherwise. This defines a local maximum ejectable grain radius $a_{d}^{M}\left(z_{\odot}\right)$, from which an absolute maximum ejectable grain radius $a_{d}^{M M}$ can be computed. Table 1 indicates $a_{d}^{M M}$ for all studied cases. This maximum is reached at the subsolar point, save for the two nuclei of kind "z1" where it occurs at about $z_{\odot}=30^{\circ}$. In these two cases, Table 1 also gives $a_{d}^{M}(0)$.

\section{Coma modelling methods}


As in paper D-I, we use Boltzman/Navier-Stokes solutions ("BE-NSE") to describe the gas coma. As stated in paper G-III, this is expected to be satisfactory for deriving a correct near-nucleus dayside dust coma, and this was indeed verified in paper D-I (see Figure 14 of D-I).

The gas distributions of the family \#205, plus \# 207D_z1 and \# 207D_z2 were described in papers G-II and G-III. The gas comae top207D and app207D are similar to the gas comae top207d and app207d of paper G-II, from which they differ only by having a ten times larger gas number density.

The DMC and DMF methods used to compute the dust distribution were described in paper D-I. The computational grids used here in both cases are those used for computing the gas flow, described in papers G-II and G-III.

Let us remind the reader that, in the DMF, one subdivides the emitted dust grains of each size into several subsets, or "fluids", in such a way that the trajectories of the dust grains of any given "fluid" do not mutually intersect (intersections between grains of differing fluids are permitted). The reason of it is that, if the trajectories of two grains of the same fluid intersect one another, at the intersection point the code sums vectorially the dust mass fluxes arriving along the two trajectories, creating a spurious "daughter" dust flux direction with an enhanced density in the direction of the center of mass of the arriving dust. However, if one dust flux is much greater than the second one, the effect is negligible, as the direction of the center of mass will coincide with that of the dominant dust flux which will thus be represented correctly, and the motion of the minority flux will be lost, without inconvenience. In most solutions presented in paper D-I, mutual dust crossings with comparable fluxes were almost always present on the antisolar axis. As a result, that axis appeared as a pencil of enhanced dust density, both in the DMC and in the single-fluid DMF solutions. This is because when there is axial symmetry the spurious "daughter" direction of enhanced density coincides with the line of mutual dust crossings, and provides the correct position of the resulting dust "pseudo-jet". The density fall-off on both sides of the axis is not represented accurately by the DMF, but this is probably a minor point. 
In the present problems, mutual dust crossings with comparable fluxes may be expected at the shocks present in the gas solution, because, due their large mass, the grains cannot turn sharply as do the gas flow lines. Owing to the simple geometry of the present nuclei, the shock patterns are relatively simple, and it is not too difficult to conjecture how to divide the emitted dust into spatially distinct subsets, in such a way that no mutual dust trajectory crossing occurs between grains of a given subset. The correctness of these subdivisions can be checked by tracing dust grain trajectories.

\section{Results}

In this section, we describe the computed dust comae, using only the DMC results, which are by definition considered correct in all cases (e.g., including the volcano-like distributions). However, one section will be devoted to a comparison of the results thus obtained with those obtained by the DMF method.

We will always describe the coma in a meridional plane: the reader must remember to rotate the distribution around the direction of the sun (the horizontal axis) to generate the 3-D coma.

As announced, we did not include most of the night side coma in the computational domain; therefore, no circumnuclear trajectory similar to those found in paper D-I can be identified in the solutions, which does not mean that such trajectories are absent.

\subsection{High production rate nuclei \#207D}

As was shown in Figure 2 of paper G-II and Figure 3 of paper G-III, the gas flow on the day-side of these four nuclei has a complex structure with multiple shocks caused by their inhomogeneity or by their asphericity. Due to the fact that the near-nucleus flow regime at such a production rate is inviscid, these gas structures are rather sharp. These gas flows can eject a rather broad range of spherical grains, extending to dm-size radii (kg masses!). We will only discuss here the smallest size $j=1$ made of submicron grains (0.062 $\mu$ m radius), and the largest size $j=7$, for which we adopted in alternance the four different grain radii 
$91 \mu \mathrm{m}, 4.2 \mathrm{~cm}, 6.2 \mathrm{~cm}$ and $9.1 \mathrm{~cm}$. Figures 3,4 and 5 present the computed dust mass density of these grains, with superimposed individual grain trajectories.

\subsubsection{Submicron to millimeter size grains}

A mere overview of Figs. 3, 4 and 5 reveals dust coma structures associated either with the surface topography features, or with the surface inhomogeneity, as were the gas structures.

Before discussing them, however, let us mention the existence of a "ministructure" located in the vicinity of the $z_{\odot} \simeq 90^{\circ}$ terminator, in all \#207D nuclei, but not visible on the present Figures, because of its small size. It was found in the previous papers G-II and G-III that, when negligible night side gas production is assumed (as here for the nuclei \#207D due to their small $\kappa$ ), gas emitted just before the terminator recondenses just beyond it, carrying with it submicron size dust grains. The effect is well visible on Fig.2 of paper D-I (trajectory emanating from $z_{\odot}=88.15^{0}$ ). Here, we find that, indeed, the submicron grains \#05 emitted from within a few degrees before the terminator fall-back within a few degrees beyond it, forming a minuscule fountain not visible on the Figures because of its too small apex (not more than $20 \mathrm{~m}$. !) This effect deserves mention because it results in a non-negligible surface bombardment, as we will discuss later. This effect is absent from the low-activity \#205 nuclei, because in these nuclei a significant gas production on the night side is postulated, which prevents gas condensation.

Let us now discuss the large coma structures.

The cavity of the "apple" nucleus produces, at all sizes, an axis-centered pencil of dust plus, at the submicron size $\# 05$, a "crease" in the isocontours (extending from $z_{\odot} \simeq 20^{0}$ at the surface to $z_{\odot} \simeq 60^{0}$ on the graduated circle). The latter is simply due to the fact that submicron dust follows closely the gas, assuming a similar distribution (compare with Fig. 2 of paper G-II); at larger sizes, grains "slip" with respect to the gas due to their larger inertia, following straight lines above $z_{\odot} \simeq 30^{\circ}$, which erases the density "crease". This slippage is also responsible for the build-up of the paraxial density peak in the coma of this nucleus, by the overlapping of grains "moving towards the axis" before crossing it, with grains moving 

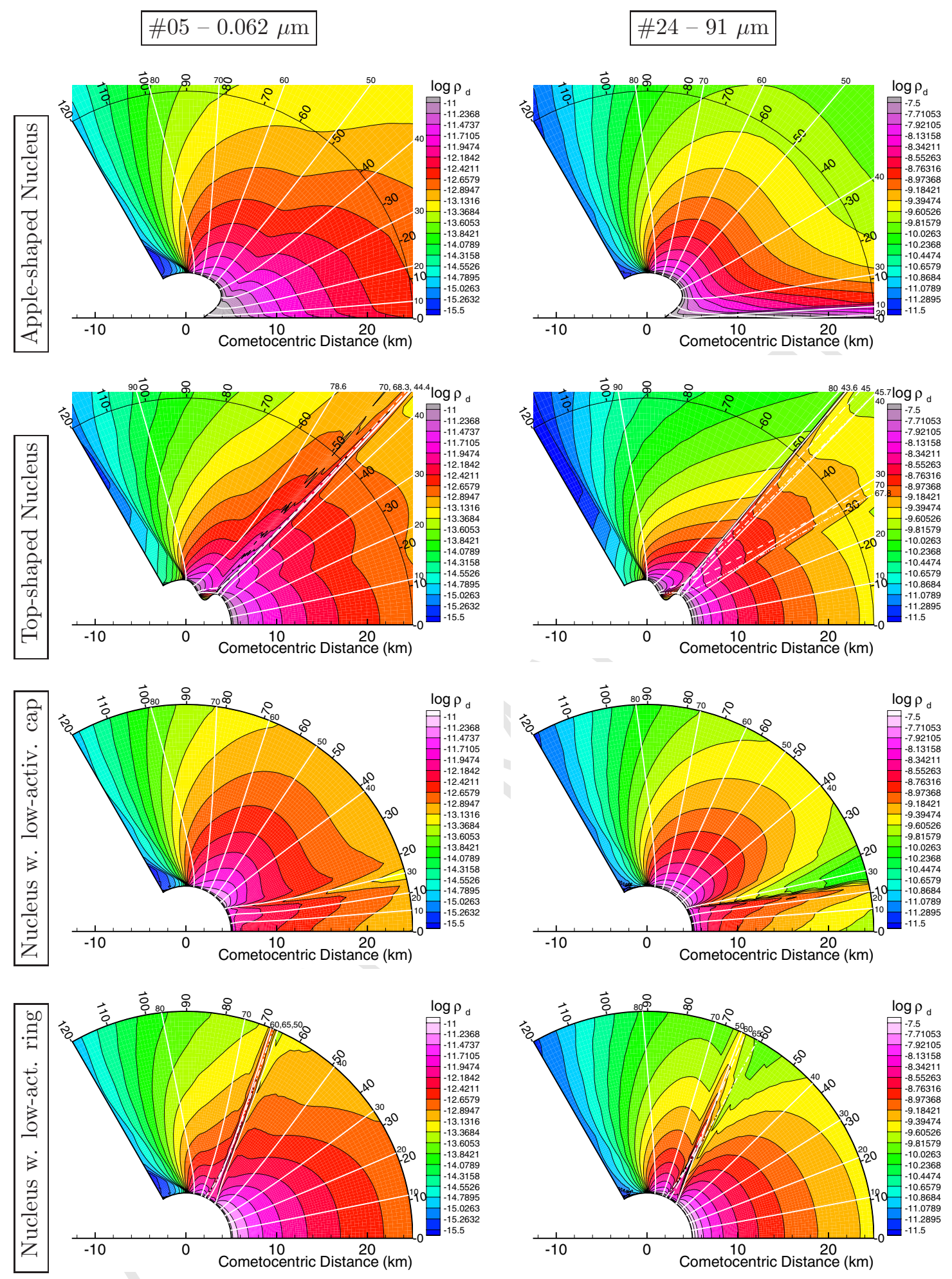

Figure 3: Highly active nuclei \#207D: trajectories (white lines) and isocontours of $\log _{10}$ (mass density, $\mathrm{kg} / \mathrm{m} 3)$ (black lines) of submicron grains \#05 (0.062 $\mu \mathrm{m}$ radius, left panels), and of micron size grains \#24 (91 $\mu$ m radius, right panels), obtained by DMC. From top to bottom: apple-shaped nucleus, top-shaped nucleus, nucleus with a weakly active cap, and nucleus with a weakly active ring. On this, and all subsequent Figures: (1) the sun is on the $+\mathrm{X}$ axis, and (2) The trajectories are labelled at their outer edge by the $z_{\odot}$ of their origin at the surface. 

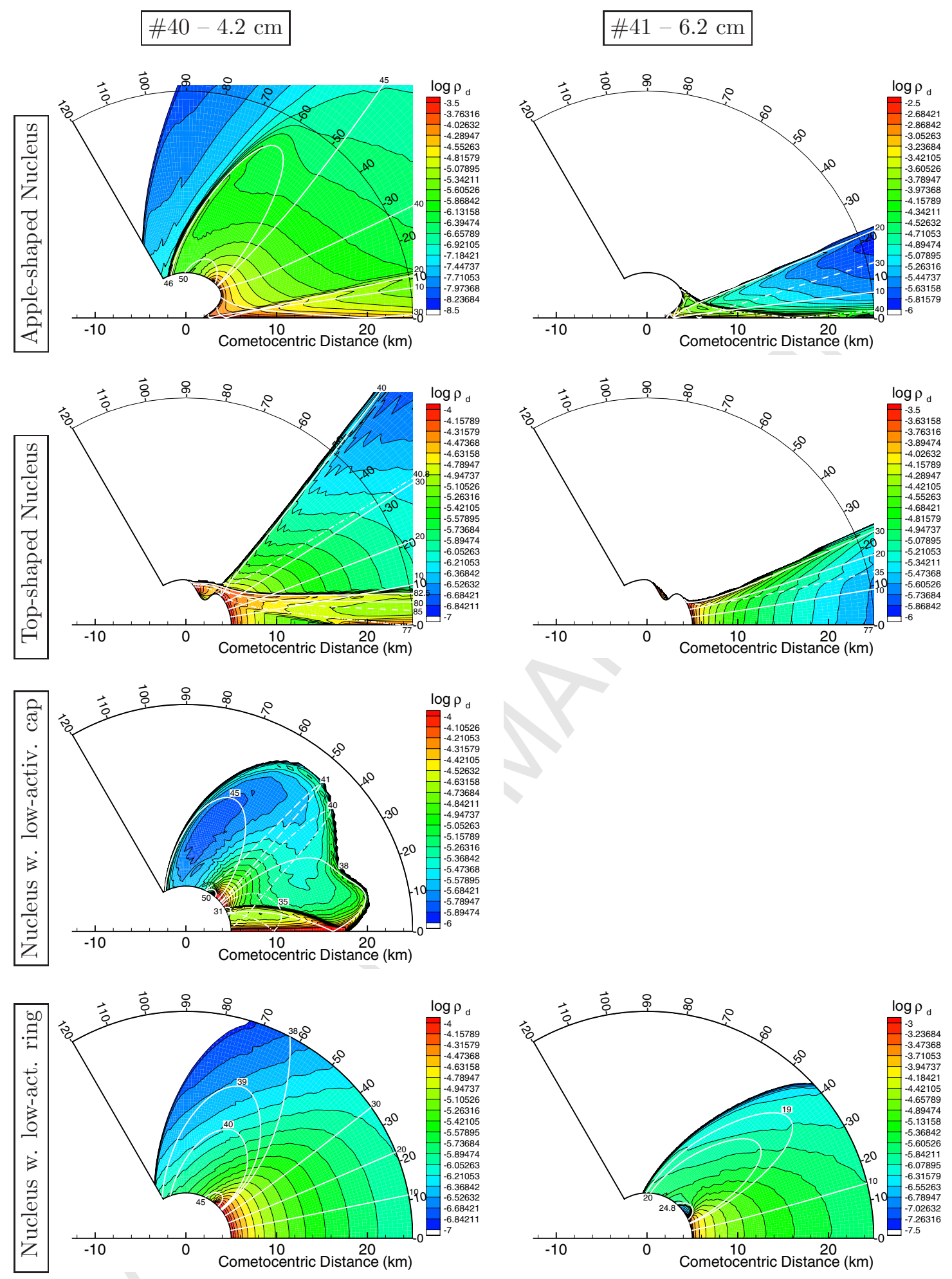

Figure 4: Highly active nuclei \#207D: isocontours (black lines) of $\log _{10}$ (mass density, $\mathrm{kg} / \mathrm{m} 3$ ), and trajectories (solid, dashed or dash-dotted white lines) of cm-size grains \#40 (4.2 cm radius, left panels) and grains \#41 (6.2 cm radius, 20 right panels), obtained by DMC. From top to bottom: apple-shaped nucleus, top-shaped nucleus, nucleus with a weakly active cap \#z1, and nucleus with a weakly active ring \#z2. The missing panel corresponds to a case where no dust ejection is possible. The sun is on the $+\mathrm{X}$ axis. 
"away from the axis" after having crossed it (see trajectories on Figs. 3 and 4). The region of overlapping increases with dust inertia, hence with dust size. This structure is similar to that appearing under axial illumination of the "bean-shaped" nucleus studied by Crifo and Rodionov (1997b). However, the cavity of the latter was an extended saddle-like feature, producing a fan-like dust distribution transverse to it. On the contrary, here, we find a narrow beam of grains because the cavity is cylindrically symmetric (pit-like). This beam is in appearance and formation process quite similar to that obtained by Knollenberg (1994) and Keller et al. (1994) from a small circular inactive region - illustrating once more the impossibility of guessing from visual inspection the origin of observed structures in dust coma images.

The cavity of the top-shaped nucleus creates at all sizes a large conical structure. At submicron size (\#05), Fig. 5 shows that it is due to a crowding of non-intersecting trajectories. At submillimeter size (\#24, Figs. 3 and 5), the density enhancement is due to the overlapping of two dust populations: the grains emitted "below" the cavity, and those emitted "above" the cavity. This can be recognized easily by inspecting the plotted grain trajectories. It resembles the effect found in the symmetry plane of the "bean shaped" nucleus, for oblique solar illumination (see Fig. 8 of Crifo and Rodionov 1997b, top left panel). In fact, the two effects (trajectory crowding, and trajectory crossings) exists at most grain sizes, but in various proportions: as the size decreases, the grain inertia also decreases and the grains follow more and more closely the gas flowlines. On the other hand, at the large size end (discussed below), due to the increasing attraction of the gravity, the region of overlapping is bent towards the symmetry axis (Fig. 4).

Coming to the inhomogeneous, spherical nuclei, we see that the weakly active ring of the nucleus \#z2 produces a narrow conical sheet of enhanced dust density, formed by the overlapping of grain trajectories from both sides of the ring. This effect is similar to that found in Crifo et al. (1995) for a related inhomogeneity. The low-activity cap of nucleus \#z1 produces a broad cone of increased density (evolving with distance towards a hollow cone), resembling that found in the mentioned problem by Knollenberg (1994) and Keller et 
al. (1994). However, here the dust density increase is not produced by trajectory overlaps, as in these references, but by a "lack of divergence" of the dust trajectories! The reason why Keller et al. (1994) obtained dust crossings, while none is found here, is that they assumed a strictly inactive circular region whereby the grain trajectories are efficiently bent towards the axis. In the present case, the circular region has a reduced, but non negligible gas production: accordingly, the grain trajectories are only confined to the vicinity of the symmetry axis, without crossing it.

To sum-up, we see that there are three basic processes leading to sharp dust density enhancements. (1) Dust trajectories piling-up: the trajectories do not intersect one another, but get closely parallel to one another. The best example is given by the "top" nucleus at size $\# 05$, creating the narrow density peak in the direction $z_{\odot} \simeq 47^{\circ}$. (2) Dust trajectory crossings (abundantly documented in the cited literature): the simplest examples being "apple" at size \#24, along the horizontal axis, and "low-activity ring" \#z2 at size \#24, near $z_{\odot} \simeq 68^{\circ}$. (3) Both processes can be combined, as with "top" at size \#24, where a broad region of crossing is bounded by two narrow regions of piling-ups, creating a spectacular V-shaped structure.

The paraxial conical beam in \#z1 is, at sizes \#05 and \#24, surrounded by a conical region of reduced dust density $\left(z_{\odot} \simeq 20^{\circ}\right)$. Its origin can only be understood by inspecting Fig. 4 of paper G-III: at the circular edge of the reduced activity cap, the gas flow lines diverge fast, thus so do the trajectories of the dust.

As found in all previous simulations of inhomogeneous nuclei (see Crifo et al., 2005b and Crifo, 2006), the coma structures produced by the present inhomogeneous nuclei are in complete contradistinction with the "intuitive" opinions often expressed in the cometary literature. Let us remind here that Whipple (1982) already pointed out (on the basis of physical probability) that dense dust pencils should best be expected to be formed by gas convergence, i.e. at the vertical of (small) inactive areas, contrary to the opposite unphysical assumption of many authors that they trace "more active" regions. 


\subsubsection{Respective appearance of the gas and dust structures}

An important question is whether the observable dust structures resemble the gas structures? Since the preceding size range is believed to dominate in the visible and IR emissions, it is well fitted for answering the question. For the aspherical nuclei, it is necessary to compare the four upper panels of Fig. 3 with the two top panels of Fig. 2 of paper G-II. It appears that the submicron dust \#05 density pattern looks similar to the gas density pattern of the "apple" and "top" nuclei. At the intermediate size dust \#24 the gas and dust density patterns of the "top" nucleus are similar, but not at all those of the "apple" nucleus. For the spherical, inhomogeneous nuclei, comparison between the four lower panels of Fig. 3 with Fig. 3 of paper G-III shows that, for the "weakly active cap" \#z1 nucleus, both size \#05 and \#24 paraxial density enhancement cones correspond to a similar cone of increased gas density. For the "weakly active ring" nucleus \#z2, the thin dust density maximum approximately coincides with the central depression in the broad enhanced gas structure created by the ring. Thus we see that there is no general rule. In all these cases, however, density increases are created by relative surface activity decreases. But it is clear that discrete surface activity increases (not considered here) can also produce coma density increases. These results cast a deep shadow on the possibility of guessing the pattern of gas production from visual inspection of dust coma images, even discarding the additional complications that they unavoidably involve a broad dust spectrum, and line-of-sight integration.

\subsubsection{Centimeter size grains}

Figure 4 presents the dust mass density distributions and individual grain trajectories for the grain sizes \#40 $(4.2 \mathrm{~cm})$ and \#41 $(6.2 \mathrm{~cm})$ around the four nuclei of the kind \#207D. It is immediately evident that the density patterns differ totally from those of the previous size range. In most cases, a large part of the computational domain is deprived from grains. All the computed distributions extend to infinity at small $z_{\odot}$, save that of the "weakly active cap" nucleus \#z1, which only produces a volcanic-like structure of surprising butterfly-like 
appearance. The "apple" nucleus (and this nucleus only) can also eject dust of size \#42 (9.1 cm radius). This ejection occurs from a very small area centered on the cavity bottom, forming a very narrow sunward cone of 3 degree half-angle (not shown on the Figures).

The unique characteristics of the very large grains distributions is mainly due to the fact that these grains are submitted to a gravitational force of the same order of magnitude (in the vicinity of the nucleus) as the aerodynamic force. This has the following two consequences. (1) The area of the nucleus over which the latter exceeds the former, allowing dust ejection, is reduced. For instance, the emission from the "weakly active cap" nucleus \#z1, at size \#40, occurs only from a small ring encircling the cap, whereas that from the "weakly active ring" \#z2, at the same size, is restricted to an axis-centered cap well inside the "weakly active ring" (where from such grains cannot be ejected). Thus, the effect of solar illumination on delineating the surface activity pattern is as strong as the effect of an heterogeneity. (2) The fraction of what we called in the paper D-I "finite trajectories" (i.e. ending by an impact into the nucleus) becomes large, up to possibly $100 \%$, in which case a closed volcano-like structure is formed as in paper D-I Figs. 5 and 12. Here, we have a volcano of this type in the case "z1" at size \#40 (Fig. 4). Notice that, even though in this case dust is emitted from the narrow ring $\left(30^{0} \lesssim z_{\odot} \lesssim 50^{0}\right)$, the occurence of many trajectory crossings complicates the "volcano" appearance, creating, in particular, an ogive-like structure inside the butterfly-like distribution, and a paraxial density maximum not unlike that described in the paper D-I (which appeared in a homogeneous nucleus). In the other cases of the present study where finite trajectories appear, only part of the dust grains return back to the surface: aspherical nuclei, and weakly active ring nucleus \#z2. The falling-back grains sometimes build-up a substantial part of the near-nucleus dust coma, merging smoothly with the escaping grains. For instance, Figure 4 shows that, in the vicinity of the "apple" and "weakly active ring" nuclei, the size \#40 grains coma is due to falling-back grains for $50^{0} \leq z_{\odot} \leq 120^{0}$; this could even be the case for part or all of the region $z_{\odot} \leq 50^{\circ}$, we cannot be sure as long as grain turning-backs may occur outside of our computational domain. Strictly speaking, this calls for a better definition of "falling-back 
grains", to the extent that some of the grains mirrored by the radiation pressure at very large cometocentric distances (e.g., $10^{4} \mathrm{~km}$ ) perhaps re-impact the nucleus. We do not attempt it here, however.

\subsubsection{Grain distributions inside surface cavities}

This paper is the first one in the cometary literature where the distribution of dust grains inside surface concavities is considered. Figure 5 shows details of the grains trajectories inside the cavities of the aspherical nuclei.

One sees that the whole shadowed cavity surface of the "top" nucleus is impacted by grains. At submicron size, the grains originate from a very small region centered on the left terminator. With increasing dust size, the impacting grains originate from a larger region beyond this left terminator. The velocity of impact increases with decreasing grain mass, reaching, for submicron grains, very high values $(300-500 \mathrm{~m} / \mathrm{s})$. We return to the properties of re-impacting grains in section 8 .

A surprising feature is the existence of trajectories grazing at very small distance the left shadowed flank of the "top" cavity before impacting its bottom. Another amazing feature associated with the presence of the "top" cavity is the existence of trajectories of grains "jumping over" the cavity and then grazing over the sunlit surface at a very small distance, before being turned outwards by the gas flow from the subsolar region.

The dust flow inside the fully sunlit apple cavity is quite different. At the submicron size $\# 05$, the grains nearly follow the gas flow lines. Only in a narrow paraxial region does the grain inertia cause some slippage of the grains with respect to the gas, resulting in mutual trajectory crossings. As their size increases, so does their inertia slippage - and the gravity force they experience; as a result, grains from higher and higher $z_{\odot}$ cross the symmetry axis under increasing angles, creating a broader and broader conical beam. At the largest size \#41 $(6.2 \mathrm{~cm})$ the grains crossing the axis near the bottom of the cavity move towards the opposite side of the cavity, and graze it before being turned back towards the axis which they cross again, thus tracing a zigzag trajectory inside the cavity. 

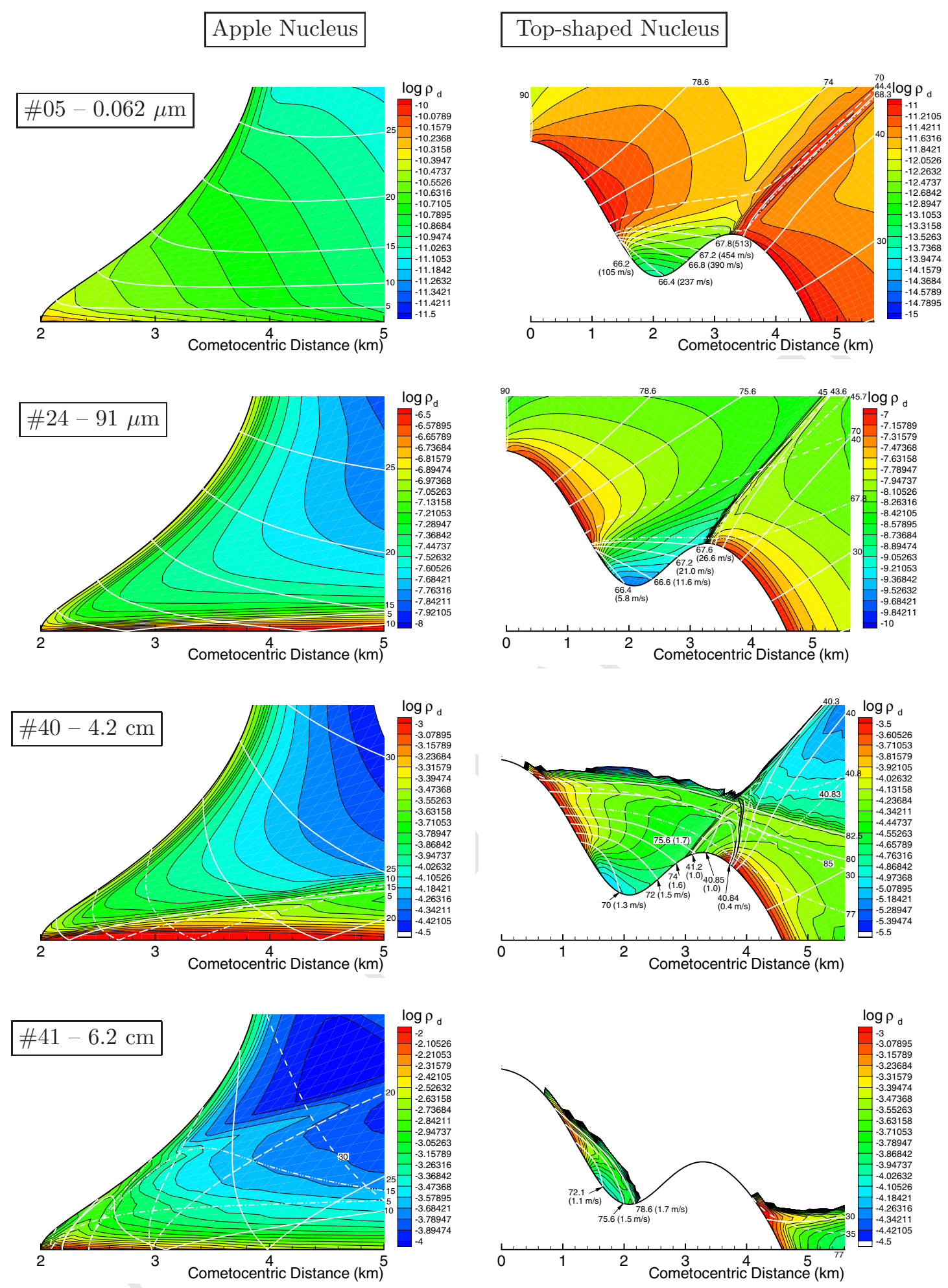

Figure 5: Highly active aspherical nuclei \#207D: isocontours (black lines) of $\log _{10}$ (mass density, $\mathrm{kg} / \mathrm{m} 3$ ) and individual trajectories (solid, dashed or dash-dotted white lines) inside the surface cavities. (From top to bottom: sizes \#05, \#24, \#40 and \#41) near the nuclei "apple" (left) and "top" (right), on a small geometrical scale. The trajectories are labelled by the solar zenith angle of their origin on the surface. The number within parenthesis indicate the grain impact velocity 
As already mentioned, dust of the largest size (\#42) (not shown) is emitted from the very bottom of the apple cavity where the aerodynamic force has a maximum and gravity a minimum. Its flow is similar to the near-axis part of the \#41 grain distribution: after grazing one cavity flank, the grains forms a very narrow beam.

\subsection{Low production rate nuclei \#205}

The question can be raised how the morphology of the near-nucleus dust coma of a given nucleus changes (at a given orientation with respect to the sun) when its heliocentric distance $r_{h}$ changes. To be sure, the coma dust mass spectrum must change, since the maximum liftable mass (at any surface point) changes considerably, and the distribution of grains of a given mass must also change, since the aerodynamic force $F_{A}$ surely undergoes changes. Could there exist some scaling law whereby the distribution of grains with radius $a_{d}^{i}$ at a distance $r_{h}^{i}$ where the total gas production is $Q^{i}$ would be similar to those of grains with radius $a_{d}^{j}$ at $r_{h}^{j}$ where the total gas production is $Q^{j}$ ?

If aerodynamic similarity holds between the gas comae at the distances $r_{h}^{i}$ and $r_{h}^{j}$, the aerodynamic force $F_{A}$ will be $\propto a_{d}^{2} Q$, hence the aerodynamic acceleration will be $\propto Q / a_{d}$. The gravitational acceleration being constant, we see that the grain trajectories will be identical if $Q^{i} / a_{d}^{i}=Q^{j} / a_{d}^{j}$ (assuming that we can neglect the radiation pressure force acceleration). Actually, Table 1 shows that the on-axis maximum ejectable masses are roughly proportional to the total production rates of nuclei \#205 and \#207D.

But does aerodynamic similarity really hold in the whole (dayside) coma? The answer would be "yes" if at both distances $r_{h}^{i}$ and $r_{h}^{j}(1)$ the whole near-nucleus gas coma was in inviscid regime, (2) on the whole nucleus surface the gas flux was strictly proportional to $Q$, and (3) the surface temperature distribution was the same. None of these three conditions is met in general, and none is met presently. On one hand, a large part of the \#205 comae is in viscous regime; it was noted, especially in paper G-II, that the gas structures become much fainter and less numerous as the production rate decreases. In particular, the shocks become smooth, because of the increase in viscosity with increasing rarefaction. On the 
other hand, sublimation is highly non-linear with respect to incident illumination, in the region of reduced illumination: hence, the gas flux at surface points with highly oblique illumination is not proportional to the total gas flux. Finally, the surface temperature changes with $r_{h}$ (however, this change is modest on the dayside if the dayside surface is icy).

When comparing the following results with the previously described high production rate ones, it will also be necessary to keep in mind that there are differences between nuclei \#205 and nuclei \#207D other than their heliocentric distance (i.e., other than their total gas production rate). (1) There is a $40 \%$ difference in nucleus size, hence in gravitational force. (2) The background gas production parameter $\kappa$ is chosen higher for the low production rate nuclei \#205. This simulates an expected relatively greater night side and shadowed area production of diffusing volatile molecules, compared to sublimating water (see papers G-II and G-III). Because the night side and shaded areas of the nuclei are relatively more active than in the case \#207D, terminator gas shocks are in general formed. (3) The weakly active ring of nucleus \#205z2 is placed at a lower $z_{\odot}$ than in nucleus \#207Dz2 (for reasons given in paper G-III).

We should therefore expect that the above $Q / a_{d}$ scaling concept is only crudely applicable here, or maybe not at all (given also that the radiation pressure is in general not negligible).

Figures 6,7 and 8 present the dust distributions and representative grain trajectories for the low production rate nuclei of the kind \#205.

It is evident from these Figures that the dust structures are neither fainter, nor less numerous than in the high activity nuclei, contrary to what was observed for the gas. This difference between gas and dust comae is clearly due to the absence of the smoothing effect of viscosity for the collisionless dust .

\subsubsection{Grains sizes $0.062 \mu \mathrm{m}(\# \mathbf{0 5})$ and $0.91 \mu \mathrm{m}(\# \mathbf{1 2})$}



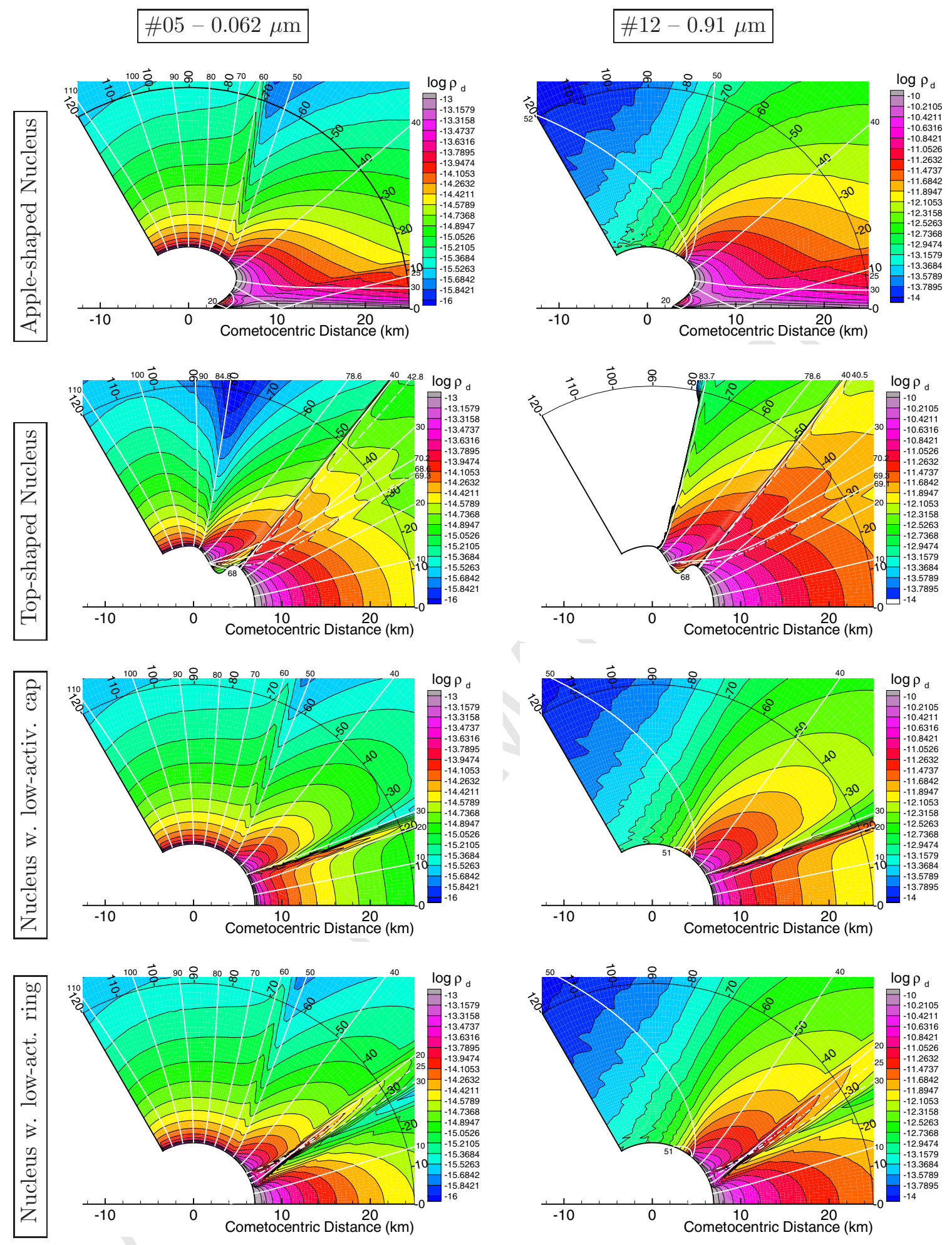

Figure 6: Low-activity nuclei \#205: isocontours of $\log _{10}$ (mass density, $\mathrm{kg} / \mathrm{m} 3$ ) (black lines) and individual grains trajectories (solid or dashed white lines) of grains \#05 (0.062 $\mu \mathrm{m}$ radius, left panels) and \#12 (0.91 $\mu$ m radius, right panels), obtained by DMC. From top to bottom: apple-shaped nucleus, top-shaped nucleus, nucleus with a weakly active cap \#z1, and nucleus with a weakly active ring $\# \mathrm{z} 2$. The sun is on the $+\mathrm{X}$ axis. 

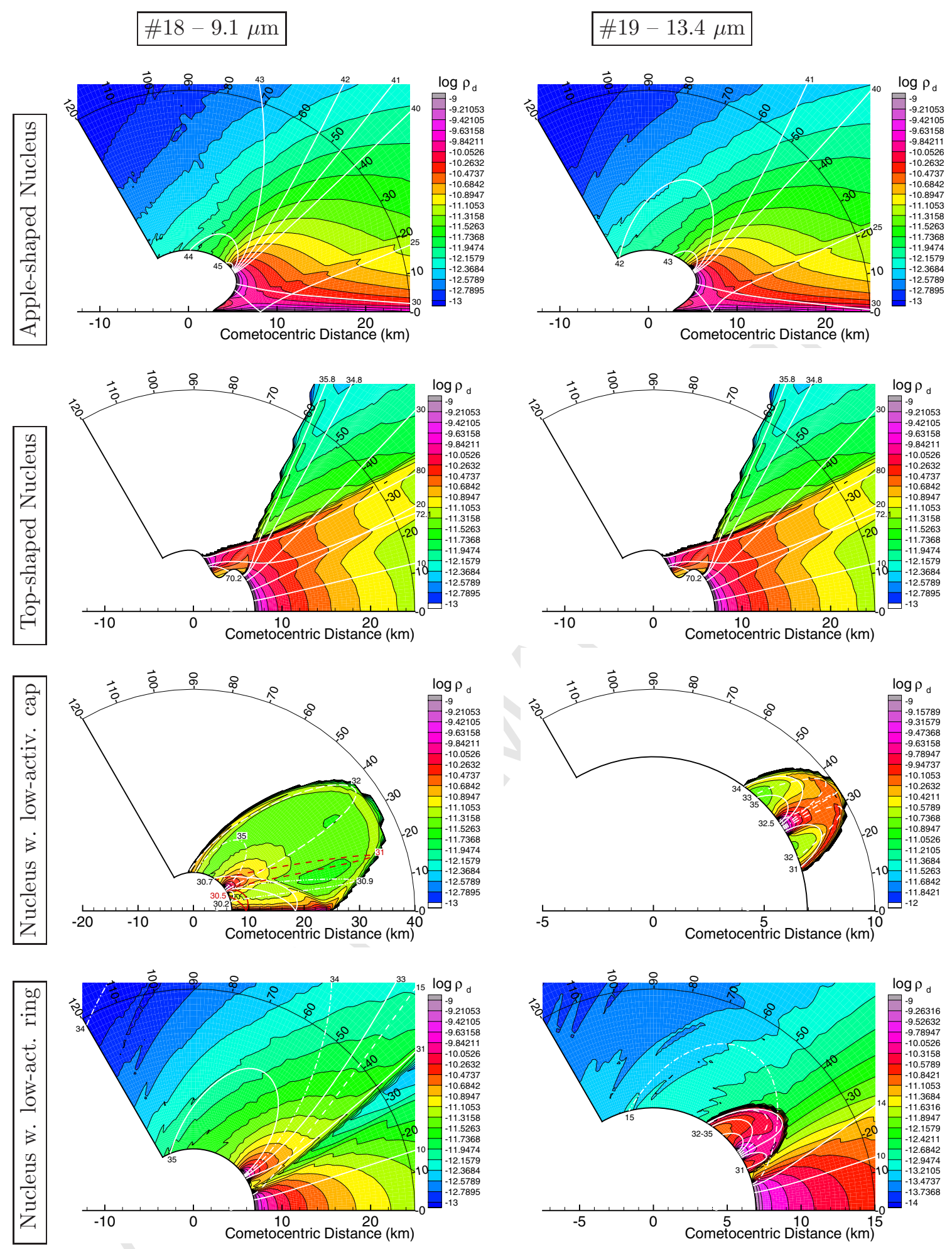

Figure 7: Low-activity nuclei \#205: isocontours of $\log _{10}$ (mass density, $\mathrm{kg} / \mathrm{m} 3$ ) (black lines) and individual trajectories (solid, dashed or dash-dotted white or red lines) of grains \#18 (9.1 $\mu \mathrm{m}$ radius, left panels) and \#19 (13.4 $\mu \mathrm{m}$ radius, right panels), obtained by DMC. From top to bottom: apple-shaped nucleus, top-shaped nucleus, nucleus with a weakly active cap \#z1, and nucleus with a weakly active ring $\# \mathrm{z} 2$. The sun is on the $+\mathrm{X}$ axis. 

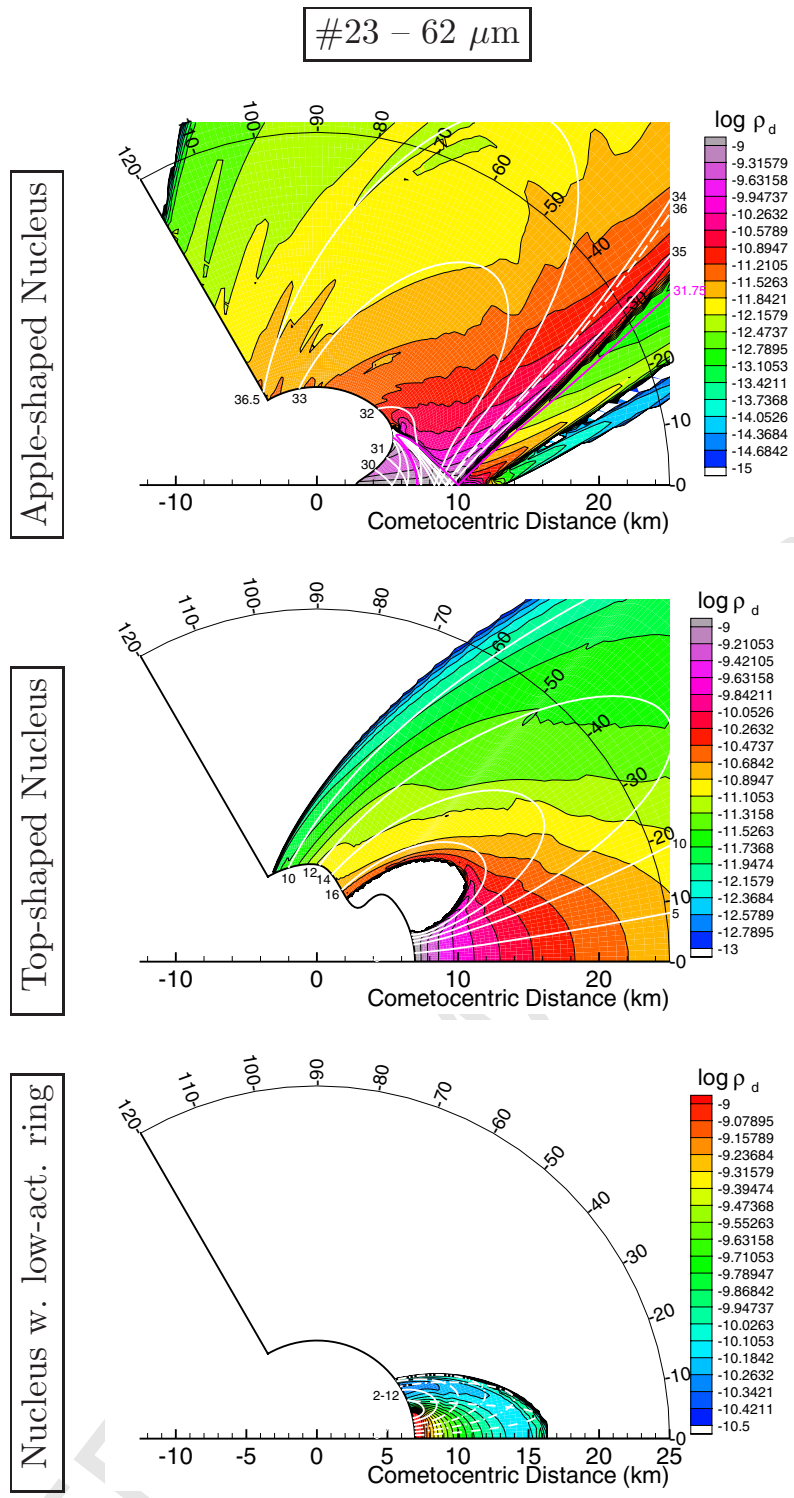

Figure 8: Low-activity nuclei \#205: isocontours of $\log _{10}$ (mass density, $\mathrm{kg} / \mathrm{m} 3$ ) (black lines) and individual trajectories (solid, dashed or dash-dotted white or red lines) of grains of size \#23 (62 $\mu \mathrm{m}$ radius), obtained by DMC. Top, left: apple-shaped nucleus. Top, right: top-shaped nucleus, Bottom: nucleus \#z2 having a weakly active ring. No grain of these sizes can be lifted off the nucleus \#205_z1 (weakly active cap). The sun is on the $+\mathrm{X}$ axis. 
On most of the day side $\left(z_{\odot}<70\right)$ the dust density distributions \#05 and \#12 resemble one another. Given that the total gas production rate ratio between the sets \#205 and \#207D is about 1000 , the preceding crude scaling suggests that this grain size range in the \#205 comae might be the equivalent, in the \#207D comae, of the range $62-910 \mu \mathrm{m}$ which includes the computed size \#24 $(91 \mu \mathrm{m})$ thus best suited for a comparison. Indeed, the present distributions do resemble those of \#24 in \#207D as tentatively expected from the scaling criterion (keeping in mind the mentioned difference in position of the weakly active ring). However, there also appear differences. Firstly, the \#05 distribution in \#205 has a high latitude "crease" associated with the gas terminator shock (at $\sim 70$ degrees) created by the mentioned postulated relatively higher shadow gas emission. Secondly, the emission at size \#05 persists at high zenith angle and in the night side, for the same reason.

\subsubsection{Grain sizes $9.1 \mu \mathrm{m}(\# \mathbf{1 8})$ and $13.4 \mu \mathrm{m}(\# \mathbf{1 9})$}

These grains (and the next ones discussed below) are too large to be lifted by the present background gas emission. The crude scaling indicates that this range corresponds, in the \#207D comae, to the range $0.91 \mathrm{~cm}-1.3 \mathrm{~cm}$ which contains none of the sizes treated here, hence no test of this scaling is possible. However, the \#18 coma (Fig. 7) clearly resembles the \#40 (4.2 cm radius) coma in the \#207D series (Fig. 4). We will see later that the apple cavity flow is nonetheless different.

With size \#19, an amazingly new structure appears near to the "top" nucleus, due to the presence of two populations: the grains ejected from a narrow region near $z_{\odot}=30^{0}$ form a fountain splashing the night side, while those emitted from beyond the cavity graze the surface to merge with the paraxial flow of grains emitted near to the axis of symmetry. It follows the formation of a large torus free of grains!

\subsubsection{Grain sizes $62 \mu \mathrm{m}(\# \mathbf{2 3})$ and $91 \mu \mathrm{m}(\# \mathbf{2 4})$}

The crude scaling indicates that this range corresponds, in the \#207D comae, to the range $6.2 \mathrm{~cm}-9.1 \mathrm{~cm}$ in \#207D which are the sizes \#41 and \#42, 
The only spherical homogeneous nucleus capable of emitting dust in this size range is that with a low active ring \#205z2, and it can emit only grains of size \#23, which form a mini volcano completely identical to the one described in paper D-I, as expected since it is confined to the vicinity of a homogeneous, spherical part of the nucleus.

The "top" and "apple" nuclei, which have a reduced mass ("top") or a reduced surface gravity ("apple" at the bottom of its cavity) both eject dust of sizes \#23 and \#24. Figure 8 shows that the "top" nucleus forms a large volcano (incompletely shown on the Figure) whose internal part differs markedly from the volcano-like flows from spherical nuclei. In particular, a dust-free region is present which can be shown to be due to the asphericity of the gravity. At size \#24, a very small size (apex at $11 \mathrm{~km}$ ) axially summetric volcano is formed, similar to that of a spherical nucleus.

The structure of the coma of these sizes near to the "app" nucleus is quite complicated, and is intimately linked to the presence of the large subsolar cavity, hence is discussed below.

\subsubsection{Grain distribution inside the surface cavities}

As stated precedingly, the flows inside the "top" cavity - when existing - resemble those found in the high activity nuclei. Such is not the case for the flows inside the "apple" cavity. By referring to paper G-II (Figure 4) one will note that in the case \#app205 the gas does not exit from the cavity, but forms a complex cellular flow inside it. Figure 9 shows enlarged isodensities and grains trajectories in this cavity. One sees that, at any size, grains are emitted only from a very small region centered on the bottom of the cavity, and from the boundary of the upper edge of the cavity. The former ones fall back onto the bottom of the cavity, forming a very small volcano with apex much smaller than the depth of the cavity. As to the grains emitted from the edge of the cavity, they divide themselves in two fractions. One fraction splashes the two flanks of the cavity (sometimes after having overflown it at a very small distance). The other fraction, escapes from the cavity after crossing the axis of symmetry. At size \#23, these escaping grains form a large-size volcano 
(only partially visible in Figure 8), while at size \#24 this fraction disappears completely.

\section{$5 \quad$ Relative influence of the applied forces}

In paper D-I, we pointed-out the need to distinguish between local and global criteria for estimating the relative role of the applied forces. In the local analysis, we declare that one of the three forces $\vec{F}_{i}^{j}(M)$ exerted at some point $M$ on a grain with size $j$ is negligible if:

$$
\left|\vec{F}_{i}^{j}\right| \leq \frac{\epsilon}{2}\left|\sum_{m \neq i} \vec{F}_{m}^{j}\right|
$$

where $\epsilon \ll 1$ denotes some smallness parameter ${ }^{2}$. This concept is useful to understand the origin of the computed grain motion. But it should by no means be used as a justification to omit consideration of such a force, for the following reasons. (1) That relation 3 holds for one dust mass does not imply that it holds for all other masses of interest; (2) that it holds at one point of a trajectory does not implies that it holds at all other points; (3) to check relation 3, one must compute the whole dust coma taking into account all three forces. What would be gained, after it, by re-doing the computation with a smaller number of forces?

Systematic investigation of Eq. 3 for all cases treated here, using the arbitrary value $\epsilon=0.1$ shows that, in the high activity cases \#207D, the solar radiation force is negligible at all sizes in the present computational domain, whereas the gravity is negligible in the whole dayside coma at grain radii smaller than $0.42 \mathrm{~mm}$; above it, as size increases, the region where the gravity is non-negligible increases from the terminator and inactive cap vicinity $\left(a_{d} \simeq 0.42 \mathrm{~mm}\right)$ to the whole dayside coma $\left(a_{d} \geq 2.9 \mathrm{~mm}\right)$. Two examples are given in the lower-left panels of Figure 10.

As with the low-activity cases \#205, the results are in agreement with what was found in Fig. 9 of paper D-I: at any size, (1) there exists a large region where none of the forces is negligible, and (2) there exists a region where the solar force is negligible but the extent

\footnotetext{
${ }^{2}$ Quite unfortunately, we forgot to indicate in paper D-I that we used (in our text and Figures) this criterion with: $\epsilon=0.1$.
} 

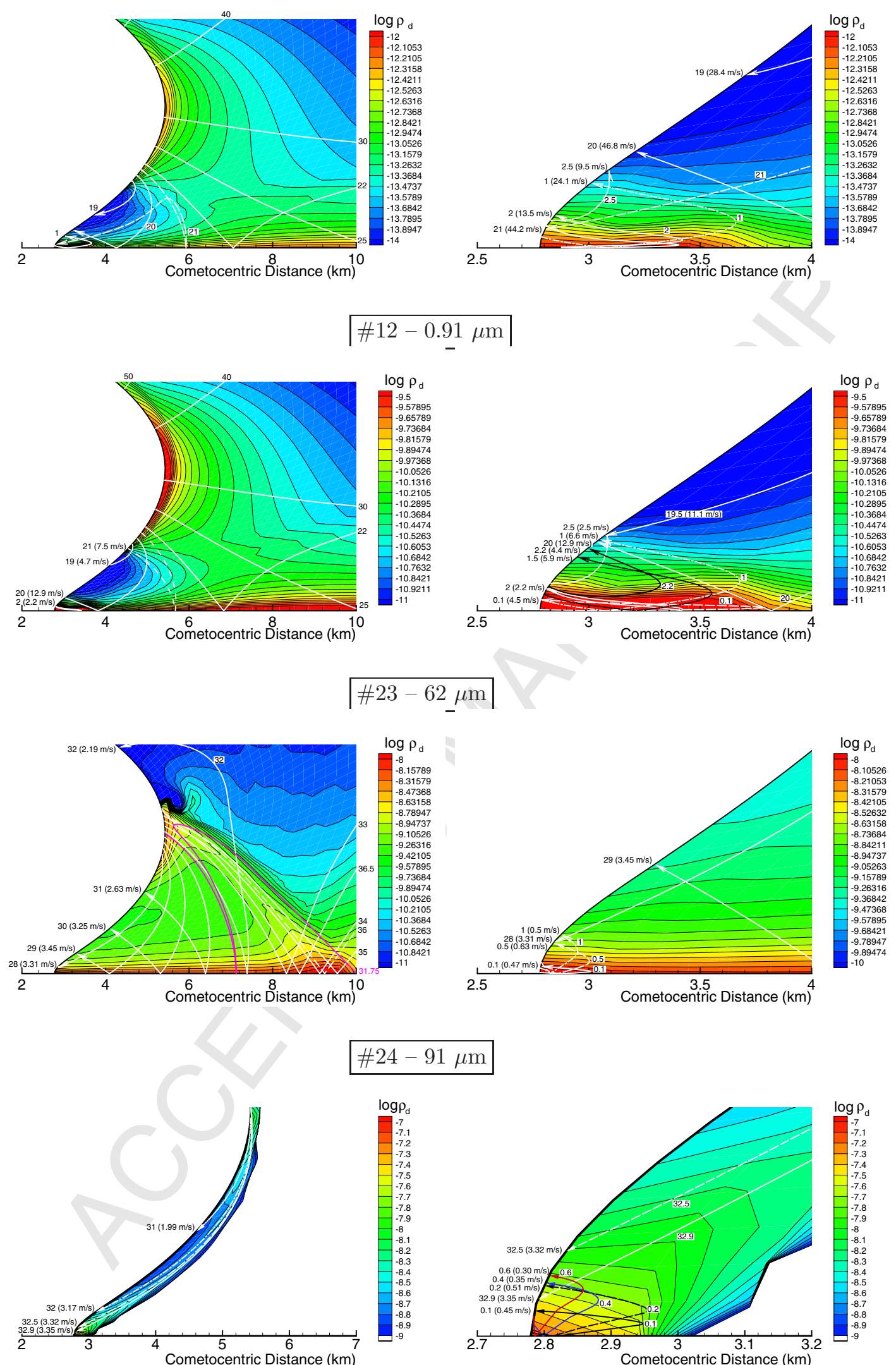

$$
\neq 24-91 \mu \mathrm{m}
$$

Figure 9: Low activity "apple" nucleus \#205: individual grain trajectories (solid, dashed or dash-dotted coloured lines) and isocontours of $\log _{10}$ (mass density, $\mathrm{kg} / \mathrm{m} 3$ ) (thin black lines) inside the cavity, on two different scales. From top to bottom, grains of size \#05, \#12, \#23 and \#24. At impact points, the solar zenith angle of grain ejection, and their impact velocity are given. 
of this region is in general small. One example is given in the upper-left panel of Figure 10. One sees that one is dealing with a case where none of the three forces is negligible in most of the domain accessible to the grains.

In a global analysis, one simply varies by some amount, one by one, the forces, and looks at the effect on the computed densities and velocities. Let us apply this method to the two preceding examples. The upper right panel of Fig. 10 compares the distribution of 9.18 $\mu \mathrm{m}$ grains from the low-activity inhomogeneous nucleus \#205z1, computed with all forces taken into account (top half-space), and computed with the radiation pressure force ignored (lower half-space). One sees how severe is the effect of this force on the motion of micronsize grains in the case of a small activity nucleus. This fact was overlooked precedingly by all authors (including us). On the other hand, the fact that this volcanic-like distribution still exists in the absence of solar force demonstrates that one is not at all dealing with a classical fountain model distribution with very small apex.

The middle-right panel compares the distribution of $6 \mathrm{~mm}$ radius grains (one gram in mass) near to the high-activity inhomogeneous nucleus \# 207Dz1, computed with the nominal gravity (upper half-space), to that computed without any gravity (lower half-space). One observes that the pattern of coma density structures has changed quite significantly in the paraxial region as well as near the terminator, due to the difference in grain trajectories.

If we remove completely the gravity from consideration at larger sizes, for the same nucleus, the change in coma structure will be greater and greater, because at such sizes large regions where from the gravity prevents emission (see Figure 4) will become active. Thus it is more instructive to study the effect of small changes in the gravity magnitude. The lower-right panel compares the distribution of $4.2 \mathrm{~cm}$ grains near nucleus \# 207Dz1, computed with the nominal gravity (upper half-space), to that computed with a density increased by $5 \%$ only (lower half-space). One sees how sensitive the heavy grain motion of such grains is to the nucleus gravity (that is, to the nucleus mass). The physical reason for it is that we are approaching the upper limit of grain ejection: the aerodynamic force cancels most of the gravity, so that the resulting force is in order of magnitude comparable 
reduced-activity-cap, Weakly active Nucleus 205z1
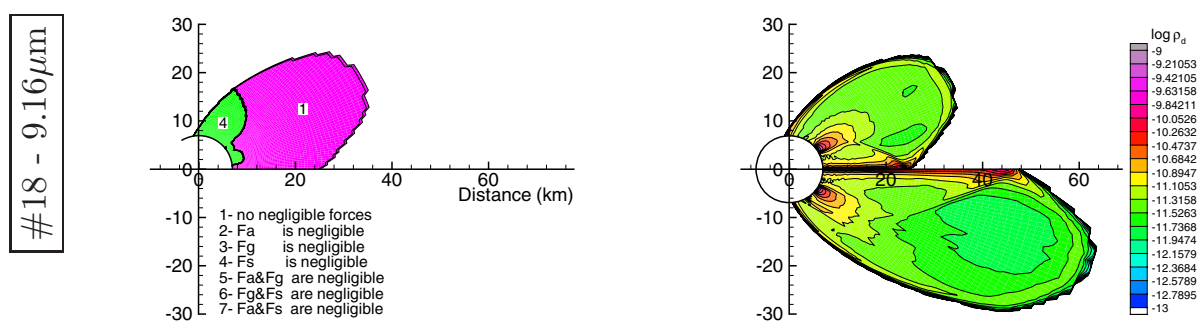

reduced-activity-cap, Highly active Nucleus 207Dz1
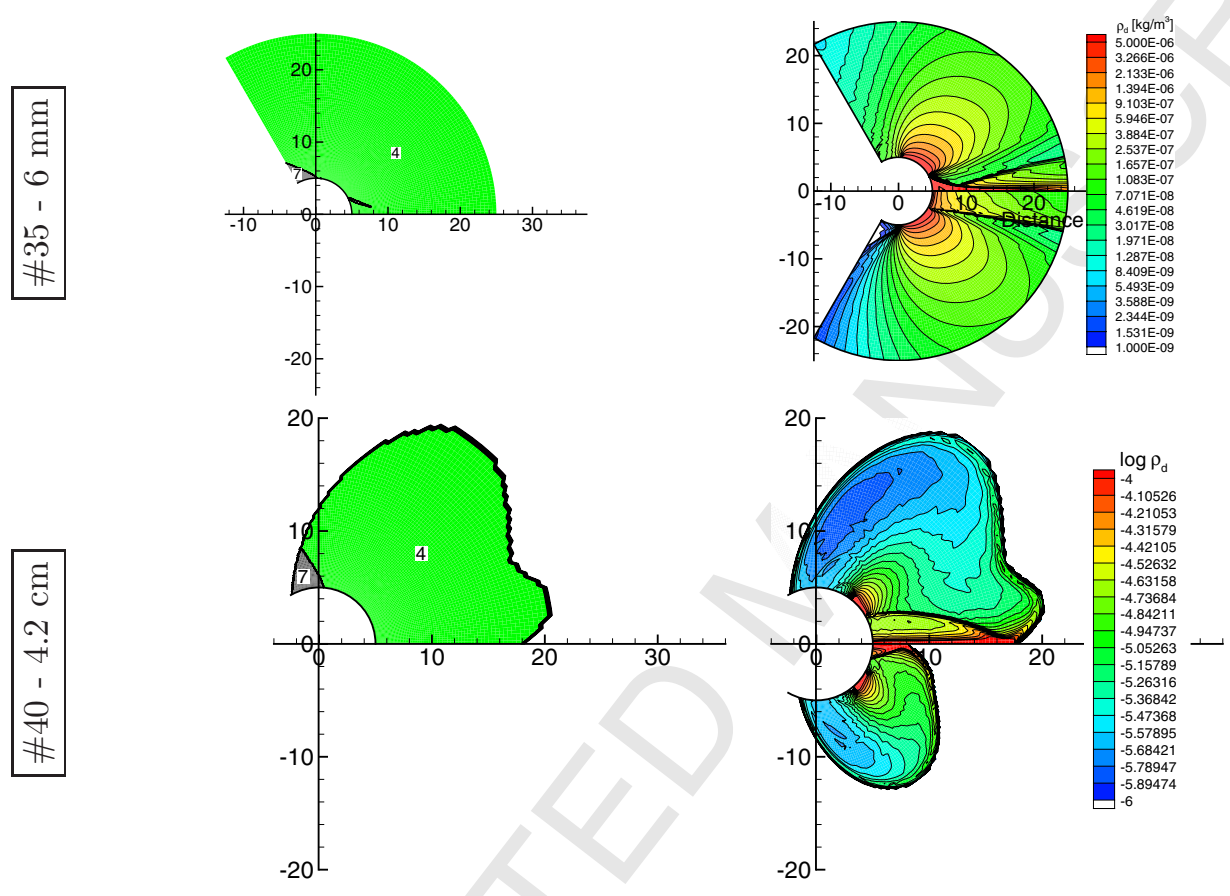

Figure 10: Sensitivity of the dust distribution to the applied forces. Left panels: Diagnostic of how many forces are negligible using the local criterion Eq. 3. Upper Right panel: computed dust density with allowance for the solar force (upper half space of the panel) and with omission of the solar force (lower half space of the panel). Middle right panel: computed dust density using the nominal gravity (upper half space of the panel) and with gravity omitted (lower half space of the panel). Lower right panel: computed dust density using the nominal gravity (upper half space of the panel) and with a gravity increased by $5 \%$ (lower half space of the panel). The two top panels refer to a micron-size grains volcano in a weakly active nucleus, the two middle panels refer to mm-size and the typ bottom panels to cm-size grains, in a highly active nucleus. The sun is on the $+\mathrm{X}$ axis. 
to a small fraction of the gravity.

We return on the influence of the gravity below.

\section{Validity of the DMF method}

One of the goals of this study was to compare coma structures computed by the DMC and DMF techniques, in the cases where both techniques are usable (this excludes for instance re-impacting grains). In order to maximize the meaning of the comparison, the same gravity fields must be used in the two techniques. For the sake of simplicity, the origin-centered $M_{n}^{\star} / r^{2}$ field was used.

For apple shaped nuclei, or weakly-active-cap nuclei, one fluid was made of the grains emitted from the apple cavity (resp. from the weakly active cap), and the second fluid included all other grains. For top-shaped nuclei, or nuclei with a weakly active ring, one fluid included the grains emitted from the subsolar cap limited by the edge of the cavity (resp. from the subsolar cap limited by the inner side of the ring), a second fluid included the grains emitted from the cavity (resp. ring) and a third included the rest of the dust (i.e., grains emitted at high $z_{\odot}$ ). We will designate the solutions based on these subdivisions as "two-fluid" and "three-fluids" solutions (acronyms $2 \mathrm{~F}$ and 3F). For reference, we also computed solutions with no subdivision into subsets: the so-called "single-fluid" solutions (acronym $1 \mathrm{~F})$.

Figure 11 compares the 1F-DMF, 2F-DMF or 3-F DMF, and DMC solutions for the top207D and app205 nuclei. These solutions are characterized by trajectory crossings (see Figs. 3 and 6). One sees, first, that, as expected on general grounds, there is a large difference between the $1 \mathrm{~F}$ and $2 \mathrm{~F}$ or $3 \mathrm{~F}$ solutions, in the region where trajectory crossings occur. More exactly, this happens because, not only crossings are present, but, also, the grain fluxes along these trajectories are comparable. In paper D-I, we showed solutions where trajectory crossings occurred, but with considerably differing grain fluxes, so that the $1 \mathrm{~F}$ solution was correct (only one trajectory providing a significant contribution to the total density). A second observation from the Figure is that the narrow $1 \mathrm{~F}$ density maximum in 
the crossing region ("top" nucleus) is right in the middle of the $3 \mathrm{~F}$ broad density maximum: this also expected, since the $1 \mathrm{~F}$ method yields the center-of-mass density distribution (see Rodionov et al., 2002). Finally, one sees that the DMC solution is in perfect agreement with the 2-F or 3F method - again, as expected. Similar conclusions could be drawn for the spherical, inhomogeneous nuclei.

\section{Influence of the gravitational field model}

The question we address here is to which accuracy must the gravity field be represented if one wants to obtain correct dust grain densities. We have not thoroughly investigated the question, but we present illustrative results to convince the reader that the question is serious, even though it is clear that there exists cases where even the omission of the gravity may be acceptable.

In the (unrealistic) case of spherical, homogeneous nuclei, this question is equivalent to asking to which accuracy the nucleus mass (or specific mass) must be known. Figure 10 has given a (possibly extreme) answer to the question - from which, by a crude scaling, we may expect that an inaccuracy of a few $\%$ will also have consequences on grains with radii of tens of $\mu \mathrm{m}$, in the low production \#205 cases.

When dealing with aspherical nuclei, the preceding question raises itself, but, in addition, that of whether any spherical approximation to the gravity is acceptable?

In first place, let us observe from Figure 2 that the difference in surface gravity between the two models may be large. Let us remind the reader that the best-fit spherical gravity is only a best-fit outside of the smallest sphere enclosing the object (Garmier and Barriot, 2001). As a consequence, the difference in maximum ejectable mass (between real value and approximating value) may be large. For instance, Table 1 shows that there is a factor 3 difference between the on-axis ejectable mass of app207D and \#207D_z2 whose masses differ by only $4 \%$ (and whose on-axis gas-fluxes are identical).

The next question is what is the effect on the coma structure of replacing the true gravity field by its spherical approximation? Figure 12 compares the trajectories and number 

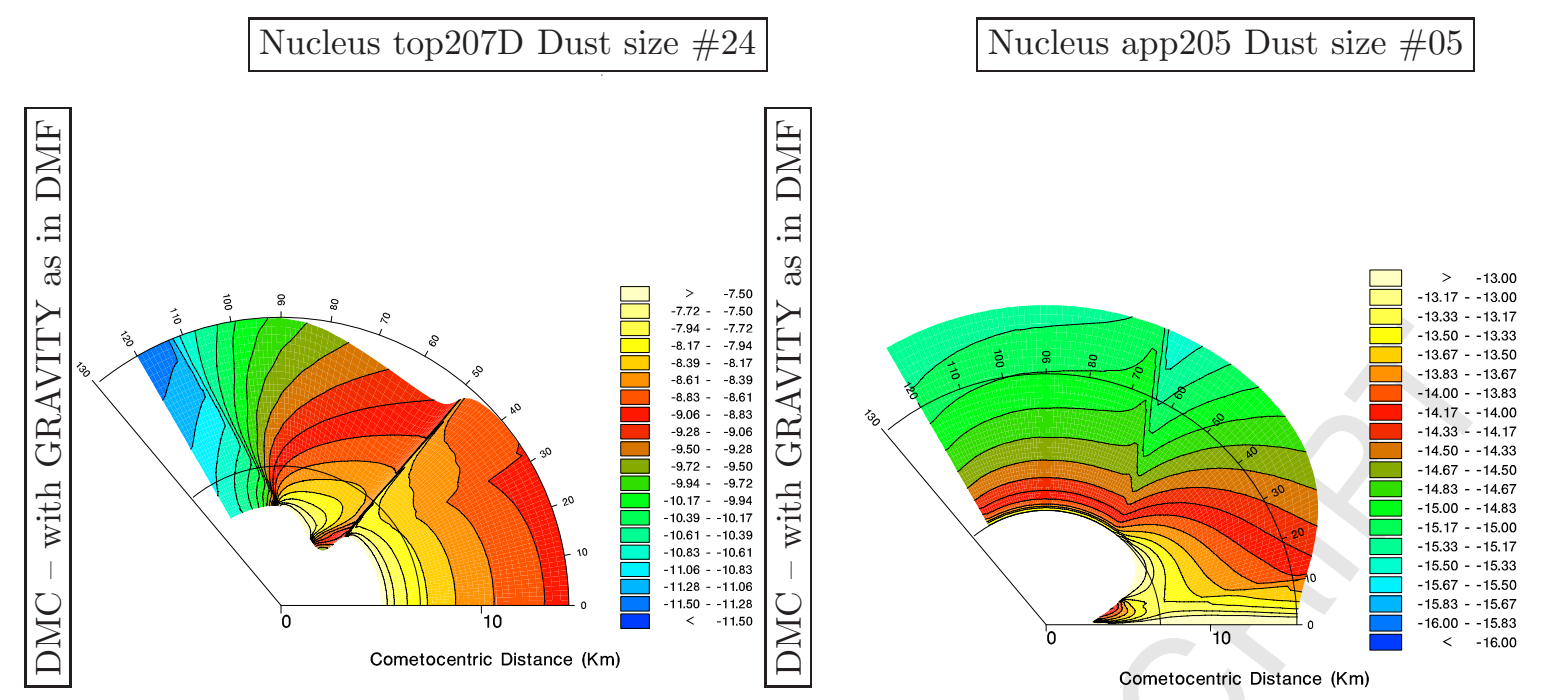

spgMtop207Db_d24mc.d Fri Feb 8 18:07:21 2008

Log10 Dust mass density (Kg m-3)
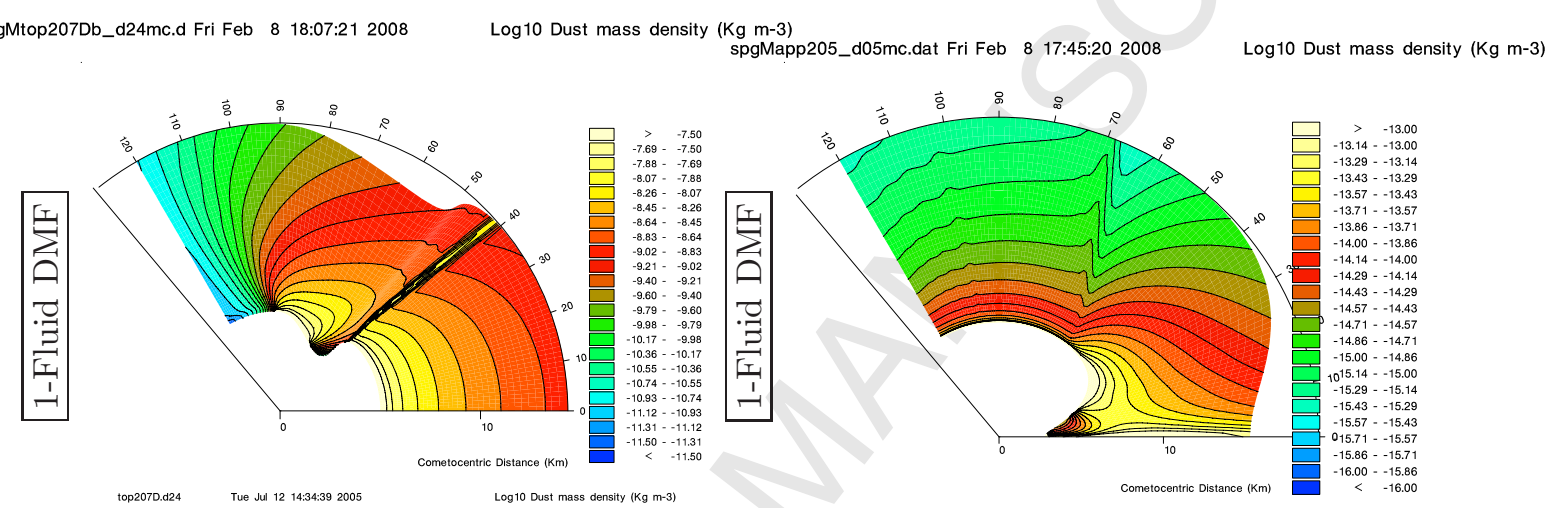
(K) $17: 45: 202008$

Log10 Dust mass density ( $\mathrm{Kg} \mathrm{m}-3)$
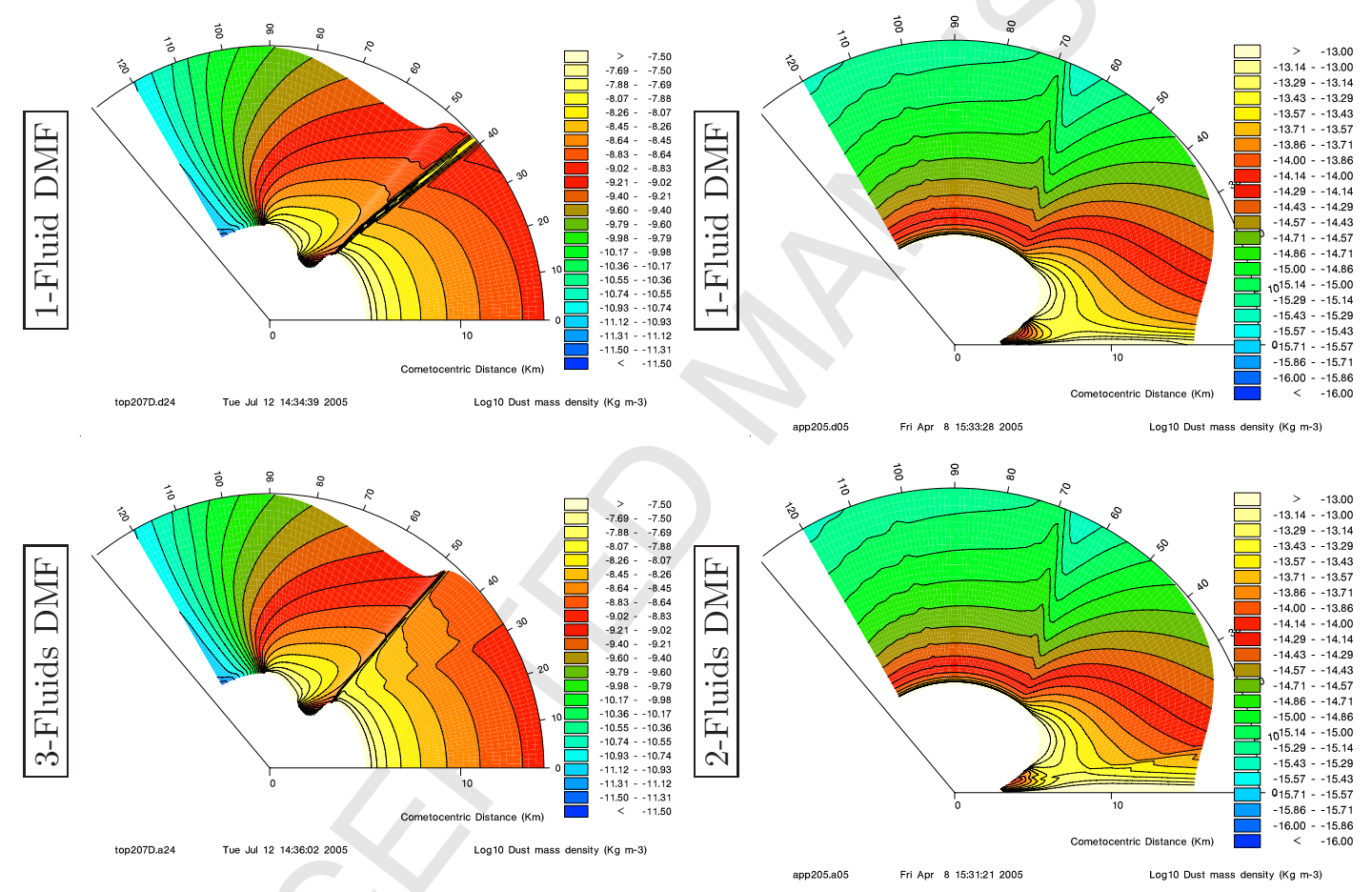

Figure 11: Comparison between the DMF and DMC modelling methods. Left: Size \#24 (0.091 mm radius) grains are considered around the high activity "top" nucleus \# top207D; Right: Size \#05 (0.062 $\mu \mathrm{m}$ radius grains) are considered around the low-activity nucleus \#app205. From top to bottom: $\log _{10}$ (mass density, $\mathrm{kg} / \mathrm{m} 3$ ) computed from DMC, using the spherical gravity field used in the DMF method; idem, computed from a single-fluid DMF method; idem, computed from a two-fluids or three-fluids DMF method. The sun is on the $+\mathrm{X}$ axis. On 40 these panels, labels with file number and date appear, for reference purposes: the reader should discard them. 


\section{DMC with BEST-FIT Spherical Gravity $\quad$ DMC with EXACT (aspherical) Gravity}
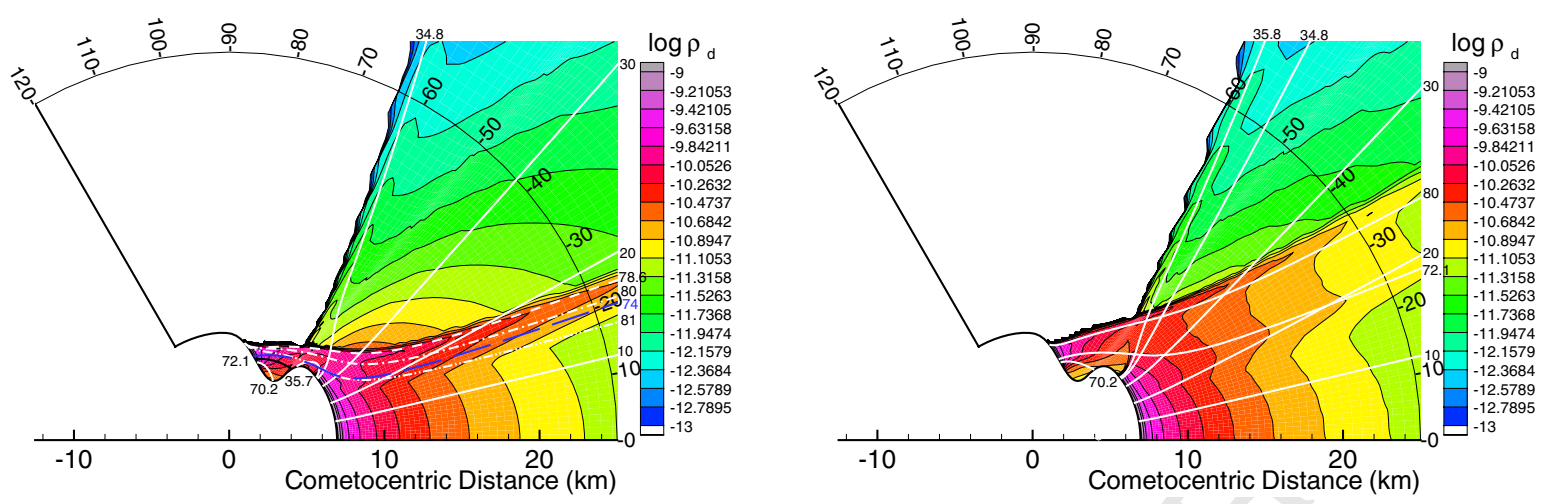

Figure 12: Influence of the gravity field model on the computed coma structure. Size \#18 (9.1

$\mu \mathrm{m}$ radius) grains around nucleus \#top205. The left panel shows the grain number density computed from DMC, using the approximate spherical gravity field used in the DMF computations. The right panel shows the grain number density computed from DMC, using the correct, aspherical gravity field. The solid or dashed white or blue lines are grain trajectories. The sun is on the $+\mathrm{X}$ axis. densities of $9.1 \mu \mathrm{m}$ radius grains around the nucleus \#top205 when (1) the exact gravity field is used, (2) the spherically symmetric field $M_{n} / r^{2}$ is used. One will notice that the difference is largest in the "pseudo-jet" region that attracts so much attention from the cometary observers. This indicates that, to correctly infer the origin of dust grains in such a case, it is mandatory to use, not the spherical approximation of the gravity, but the real gravity. This is surely a problem for a cometary rendez-vous mission, because in such a mission the gravity field is usually derived by a multipolar expansion fit to orbital data. As reminded in Garmier and Barriot (2001), such an expansion does not necessarily converge in the immediate vicinity of the surface of the nucleus. Hence how to infer the real gravity field in, e.g., cavities, is an open question. 


\section{Properties and implications of the falling-back grains}

It should be clear, in first place, that the present study does not predict the emission from any given point of any given nucleus of grains with any given mass. Therefore, it cannot predict the existence of falling-back grains. However, as soon as coma or tail observations guarantee the ejection of some kind of grains, then the present model will enable one to figure-out whether grains of this kind will re-impact the surface. This assessment is important in two respects: (1) it helps figuring-out whether the overall shape, and the composition of the surface layer of the nucleus, are genuine or are the result of impact reprocessings; (2) it will allow estimates of the dust flux to be expected on a landed probe (in first place, on the Rosetta mission lander).

Figures 13 and 14 show, respectively, the flux and velocity of the falling-back grains identified in the present study. A mere glance on the former one indicates that: (1) on highly active nuclei, fall-backs on sunlit surfaces are only due to very large grains - near to the threshold of ejection - while areas in shadow (including the night side) receive much higher fluxes, and from grains of many sizes; (2) on weakly active nuclei, a dominant part of the surface is susceptible to be bombarded, and by grains of many sizes; this bombardment is especially intense in surface cavities, where all grain sizes take part in the bombardment.

To allow a better evaluation of the significance of the re-impacting fluxes $F^{-}$, Table 3 compares the peak values $\left[\mathrm{F}^{-}\right]_{\max }$ of these fluxes (over the surface, and taking all sizes into account) to the upward fluxes $F^{+}$at the point and grain size for which $\left[F^{-}\right]_{\max }$ is obtained. One sees that, for high activity nuclei, $\left[F^{-}\right]_{\max }$ is due to submicron grains, and occurs at the terminator due to the effect we mentioned at the beginning of subsection 4.1.1; at these points, the upward flux is negligible. For low-activity nuclei, $F^{-}$peaks inside the cavities of the aspherical nuclei, where it is high and due to submicron grains, or peaks inside the reduced activity areas of the inhomogeneous, spherical nuclei, where it is small and due to micron-size grains. The upward flux is negligible with respect $\left[\mathrm{F}^{-}\right]_{\max }$, 


\section{HIGH ACTIVITY \#207D}
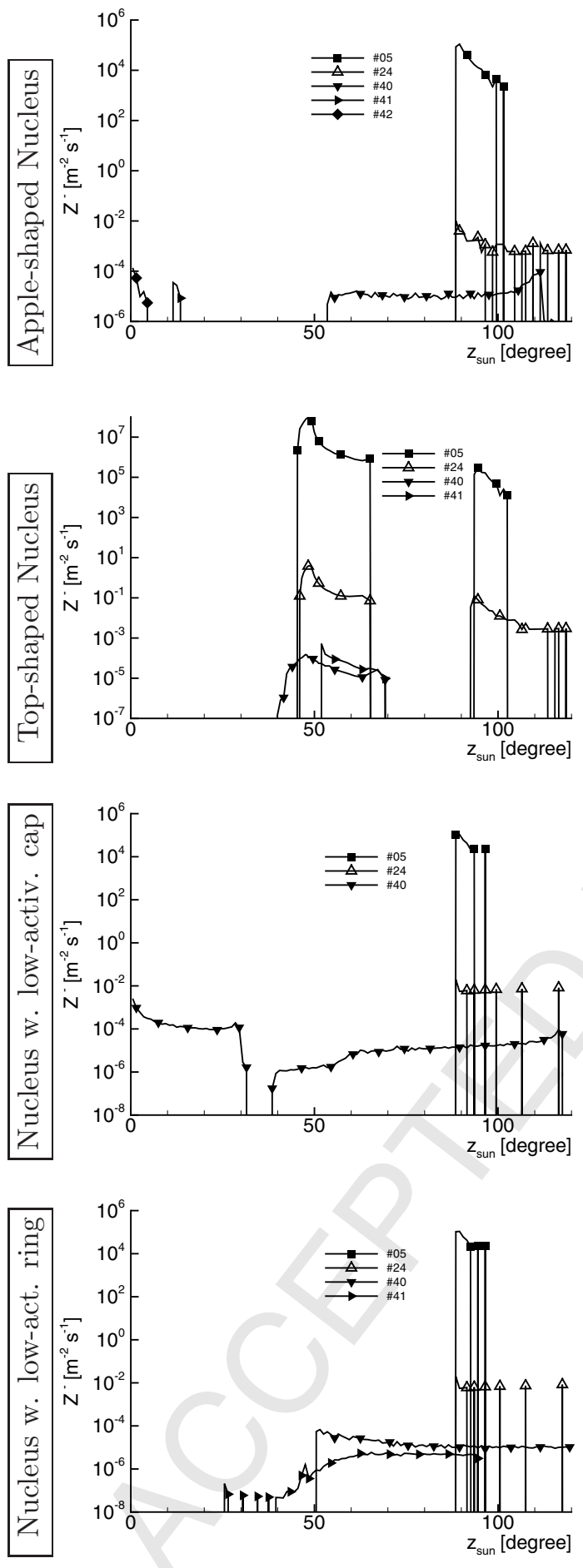

\section{LOW ACTIVITY \#205}
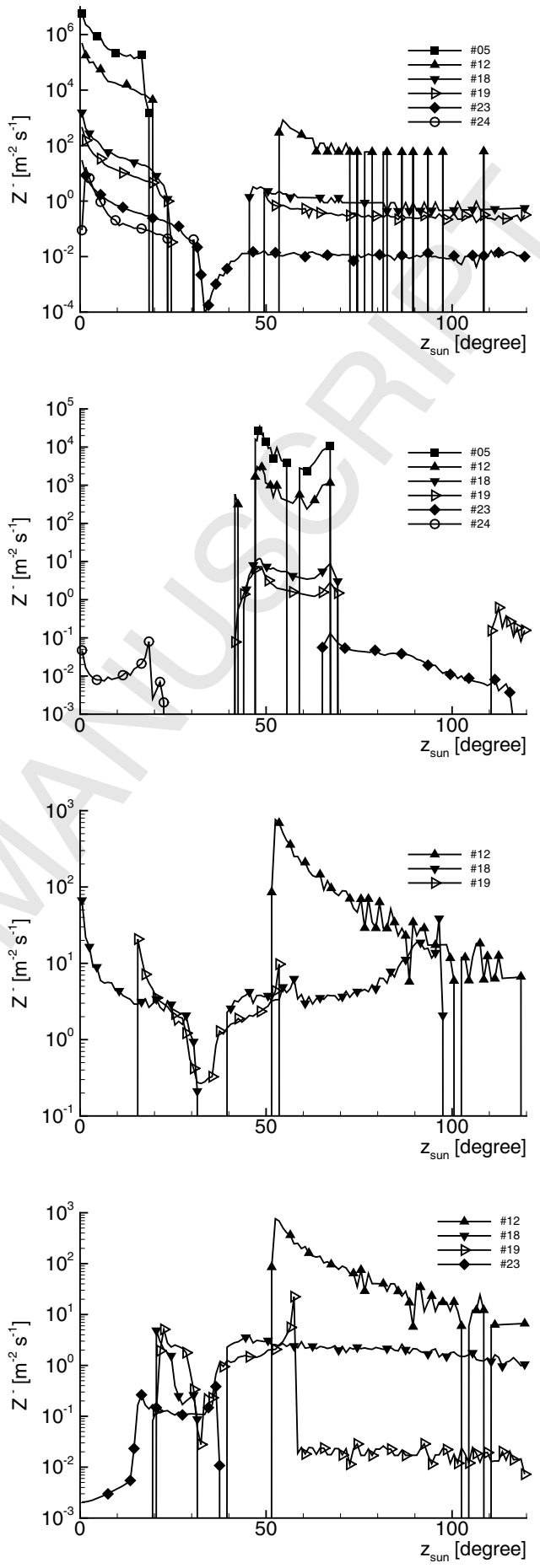

Figure 13: Flux of falling-back particles $Z^{-}$as a function of position on the surface. From top to bottom: nuclei of kinds "app", "top", "z1", "z2". Left column High activity case \#207D; Right column Low activity case \#205. The different curves correspond to different grain sizes, as indicated. Notice their differing vertical scales. The "spiky" appearance of some parts of the \#12 curve is statistical noise. The symbol $z_{\text {sun }}$ designates the solar zenith angle $z_{\odot}$. 
excepting the case of the "apple" cavity, where it is of the same order of magnitude. Thus we see that, in all cases, there is significant short-term reprocessing of parts of the surface due to grain fallbacks. When the nucleus rotates, the reprocessed area will in general move on the surface, so that the effect likely becomes global. The reprocessing is not necessarily maximal where the falling-back flux is maximal, as shown by Table 3 which indicates the peak rate of area coverage by falling-back grains $\left[\pi a_{d}^{2} F^{-}\right]_{\max }$ (in fraction per second). We see, for instance, that the region $z_{\odot} \simeq 51^{0}$ of the surface of the highly active spherical nucleus 207D_z1 can be covered in one day with a layer of size \#42 grains (i.e., a layer of $8 \mathrm{~cm}$ thickness!). The cavities of the highly active nuclei app207D and top207D can, similarly, be covered with a layer of submicron dust in a few days. Of course, the rotation will probably increase the times for complete coverage, but will as well increase the extent of the covered areas. Also, one must add to the above rates, smaller but still significant rates from grains of other sizes.

One can ask whether the energy flux due to the above impacts is significant for the nucleus (e.g., by increasing its gas production locally). We found that this flux is always less than $10^{-4}$ of the postulated energy flux assumed to arrive at the surface (solar plus thermal conduction). It is therefore unlikely that with any conceivable dust spectrum this energy flux will be significant.

The importance of the falling-back effect raises the question of its consequences for a landed probe.

Since, owing to the nucleus rotation, the probe will sample a large range of solar zenith angles, it will probably pass through areas of significant bombardment, and its space-facing surfaces will, accordingly accumulate dust of many different sizes (there is little possibility of clean-up for dust deposited on a probe). As the comet moves towards perihelion (conditions presumably comparable to \#207D), the absolute value of the bombarding flux will not increase dramatically, but the rate of coverage will increase, because the size of the fallingback grains will increase. These existence of these effects is independent from our arbitrarily (although reasonably) selected dust spectrum, since to avoid dust deposition one has to 


\begin{tabular}{|l|lll|llll|}
\hline Nucleus & Size & $a_{d}$ & $z_{\odot}$ & $V^{-}$ & $F^{+}$ & {$\left[F^{-}\right]_{\max }$} & {$\left[\pi a_{d}^{2} F^{-}\right]_{\max }$} \\
& index & $(\mathrm{m})$ & $(\mathrm{deg})$. & $(\mathrm{m} / \mathrm{s})$ & $\left(\mathrm{m}^{-2} \mathrm{~s}^{-1}\right)$ & $\left(\mathrm{m}^{-2} \mathrm{~s}^{-1}\right)$ & $\left(m^{2} / \mathrm{m}^{2}\right) s^{-1}$ \\
\hline \hline app207D & $\# 05$ & $6.2 \mathrm{e}-8$ & 90 & 11. & 0. & $1.06 \mathrm{e}+5$ & $1.26 \mathrm{e}-9$ \\
& $\# 42$ & $9.1 \mathrm{e}-2$ & 0 & 0.21 & $2.62 \mathrm{e}-5$ & $1.35 \mathrm{e}-4$ & $3.58 \mathrm{e}-6$ \\
\hline top207D & $\# 05$ & $6.2 \mathrm{e}-8$ & 48.5 & 500. & 0. & $9.37 \mathrm{e}+7$ & $1.13 \mathrm{e}-6$ \\
& $\# 41$ & $6.2 \mathrm{e}-2$ & 52.3 & 1.7 & 0. & $4.77 \mathrm{e}-4$ & $5.76 \mathrm{e}-6$ \\
\hline 207D_z1 & $\# 05$ & $6.2 \mathrm{e}-8$ & 90 & 8.7 & 0. & $1.07 \mathrm{e}+5$ & $1.29 \mathrm{e}-9$ \\
& $\# 40$ & $4.2 \mathrm{e}-2$ & 51.3 & 2.1 & 0. & $2.57 \mathrm{e}-3$ & $1.42 \mathrm{e}-5$ \\
\hline 207D_z2 & $\# 05$ & $6.2 \mathrm{e}-8$ & 90 & 8.7 & 0. & $1.07 \mathrm{e}+5$ & $1.29 \mathrm{e}-9$ \\
& $\# 40$ & $4.2 \mathrm{e}-2$ & 51.0 & 1.11 & 0. & $6.15 \mathrm{e}-5$ & $3.41 \mathrm{e}-7$ \\
\hline \hline \multirow{2}{*}{ app205 } & $\# 05$ & $6.2 \mathrm{e}-8$ & 0 & 37 & $5.12 \mathrm{e}+6$ & $5.70 \mathrm{e}+6$ & $6.88 \mathrm{e}-8$ \\
& $\# 12$ & $9.1 \mathrm{e}-7$ & 0 & 11. & $3.78 \mathrm{e}+5$ & $4.93 \mathrm{e}+5$ & $1.28 \mathrm{e}-6$ \\
\hline top205 & $\# 05$ & $6.2 \mathrm{e}-8$ & 48.2 & 25. & 0. & $3.25 \mathrm{e}+4$ & $3.92 \mathrm{e}-10$ \\
& $\# 24$ & $9.1 \mathrm{e}-5$ & 18.6 & 1.2 & 0. & $7.50 \mathrm{e}-2$ & $1.95 \mathrm{e}-9$ \\
\hline & $\# 12$ & $9.1 \mathrm{e}-7$ & 52.8 & 0.22 & 0. & 740. & $1.93 \mathrm{e}-9$ \\
& $\# 18$ & $9.1 \mathrm{e}-6$ & 0 & 1.5 & 0. & 66.4 & $1.73 \mathrm{e}-8$ \\
\hline & $9.1 \mathrm{e}-7$ & 52.8 & 0.22 & 0. & 741. & $1.93 \mathrm{e}-9$ \\
& $1.3 \mathrm{e}-5$ & 57.4 & 2.8 & 0. & 21. & $1.18 \mathrm{e}-8$ \\
\hline
\end{tabular}

Table 3: Properties of the falling-back dust fluxes. For each nucleus, the first line indicates the dust size and solar zenith angle $z_{\odot}$ where the largest downward flux $\left[F^{-}\right]_{\max }$ is found, and the second line indicates the dust size and $z_{\odot}$ where the largest rate of surface coverage $\left[\pi a_{d}^{2} F^{-}\right]_{\max }$ is found. The format $u e \pm v$ is used for $u \times 10^{ \pm v}$. 
HIGH ACTIVITY \#207D
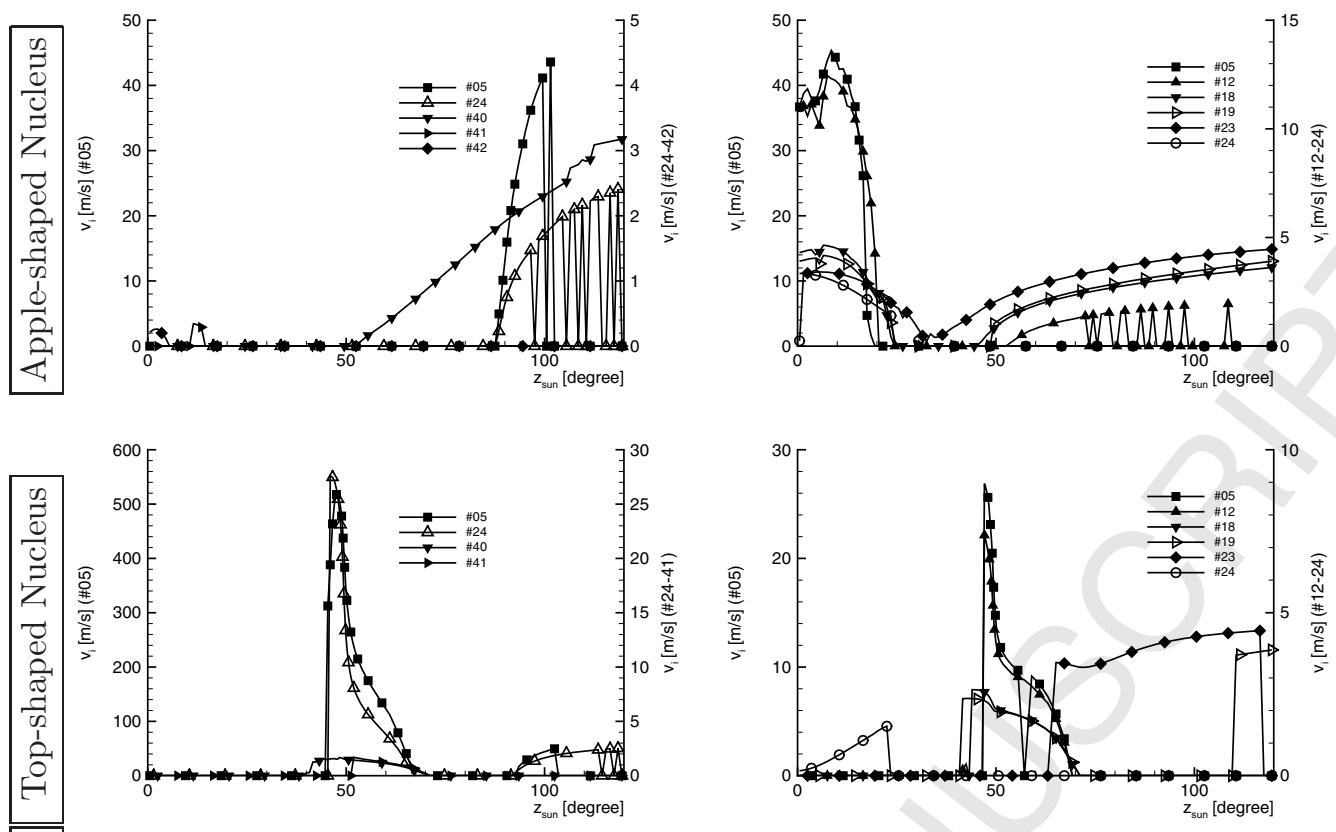

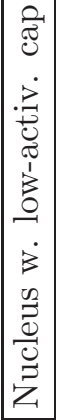
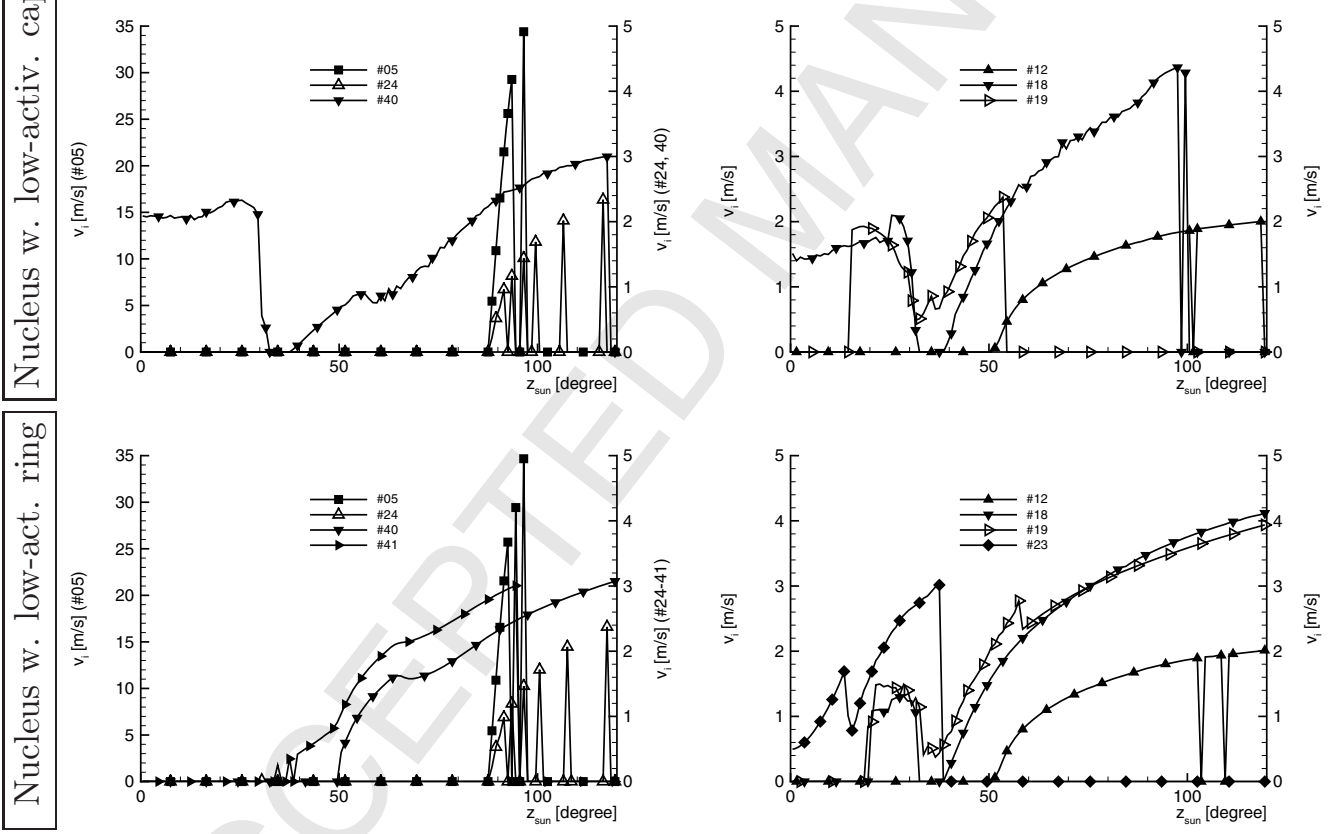

Figure 14: Impact velocity of falling-back particles as a function of position on the surface.

From top to bottom: nuclei of kinds "app", "top", "z1", "z2". Left column high activity nuclei \#207D; right column: low activity nuclei \#205. The different curves correspond to different grain sizes, as indicated. The values relative to size \#05 (when present) should be read on the left vertical scales, those relative to greater sizes must be read on the right vertical scales. The 46

"sawtooth" appearance of some \#12 curves is satistical noise. The symbol $z_{\text {sun }}$ designates the solar zenith angle $z_{\odot}$. 
make the impossible assumption $\chi^{s} \equiv 0$. But, of course, the numerical values characterizing these effects (e.g. mass deposition rates) are linked to our postulated dust size spectrum, i.e., our adopted values of $\chi^{s}$. They can be increased or decreased by other assumptions concerning this spectrum. The reader can do his own estimate by choosing his own $\chi^{s}$ values, and scaling our numbers in proportion to $\chi^{s}$.

The impact velocities, which control the impact damage to the probe, are, for the \#205 case, reasonably low at all sizes (at most a few $\mathrm{m} / \mathrm{s}$ ) excepting the cavities, where they can reach tens of m/s. As the comet moves towards perihelion, Figure 14 indicates a general increase of the impact velocities, but with varying magnitudes. For instance, in the "apple" nucleus, there is at most a $10 \%$ increase, whereas in the cavity of the "top" nucleus, there is a factor 20 increase. If any conclusion can be drawn as of now, it is to avoid landing in cavities. We already arrived at it in our studies of the gas flow, for other reasons (avoidance of frost deposition).

Coming back to the future Rosetta landing, let us observe that the target nucleus will be $\simeq 3$ times smaller than our \#205 nucleus, and is expected to have a gas production rate dominated by $C O$ and in the range 1 to 3 times that of the \#205 nucleus. If a scaling of the kind advocated above could be used, it would locate the bombardments (outside of cavities) in a dust size range increased by a factor 45 to 120 , as compared with case \#205, i.e., for the low production case, the range of radii $45 \mu \mathrm{m}$ to $4.5 \mathrm{~mm}$, and, for the high production case, the range of radii $0.12 \mathrm{~mm}$ to $12 \mathrm{~mm}$. We do not venture to make additional speculations here since, on one hand, an approximate shape of the target comet nucleus (of course different from those used here) has been derived by Lamy et al. (2007); on the other hand, it is expected that the analysis of images of the comet dust trail and tail will yield precise informations on the dust size spectrum. A dedicated detailed model of the near-nucleus dust coma of this comet should therefore become possible in the near future.

\section{Limits of the steady-state approximation}


In the present model, the applied forces are assumed time-independent (in a nucleusattached frame), and no inertia force is considered. But real nuclei rotate, where from, in a nucleus-attached frame, one must add a rotational inertia force, and the sun direction changes, which renders the aerodynamic force and solar radiation pressure force timedependent. These effects will significantly affect the properties of the re-impacting grains if their transit time from emission to impact is a sizable fraction of the nucleus rotation period. To have a feeling of when this occurs, Table 4 indicates the range of transit times $\Delta t$ for the re-impacting trajectories found in the present study. These times range from 1 to 10 hours, whatever the activity and dust size are, even though for the low-activity case they are more frequently of a few hours, while in the high activity case they are more frequently in the 10 hour range. Thus, in nuclei rotating with periods of a few days or faster, the rotation should be expected to have a strong effect. This will be investigated in the future, at least in a few cases - because one should expect that the transit times also depend upon the nucleus mass, i.e., upon its characteristic size and its specific mass, making a thorough study very cumbersome, and perhaps not very useful.

It is worth recalling also here that our assumption of the presence of large non-ejectable stones in the weakly active areas implies a long-term evolution of these areas towards total inactivity.

\section{Conclusion}

Before recalling the conclusions of the present study, let us make a general remark concerning their relevance. They are based (as those of paper D-I) on deliberately simplified benchmark representations of cometary nuclei and dust. Thus they are applicable to any real observation, but in a carefully thought manner. That is, the physical processes identified here hold in any real comet, but other processes deliberately omitted here, for instance, the inertia and tidal forces, or the non-sphericity of grains, or the presence of volatile fractions inside them, should as well be taken into consideration when interpreting observations of real dust comae. Such effects - who knows? - could counter-act or alter the presently 


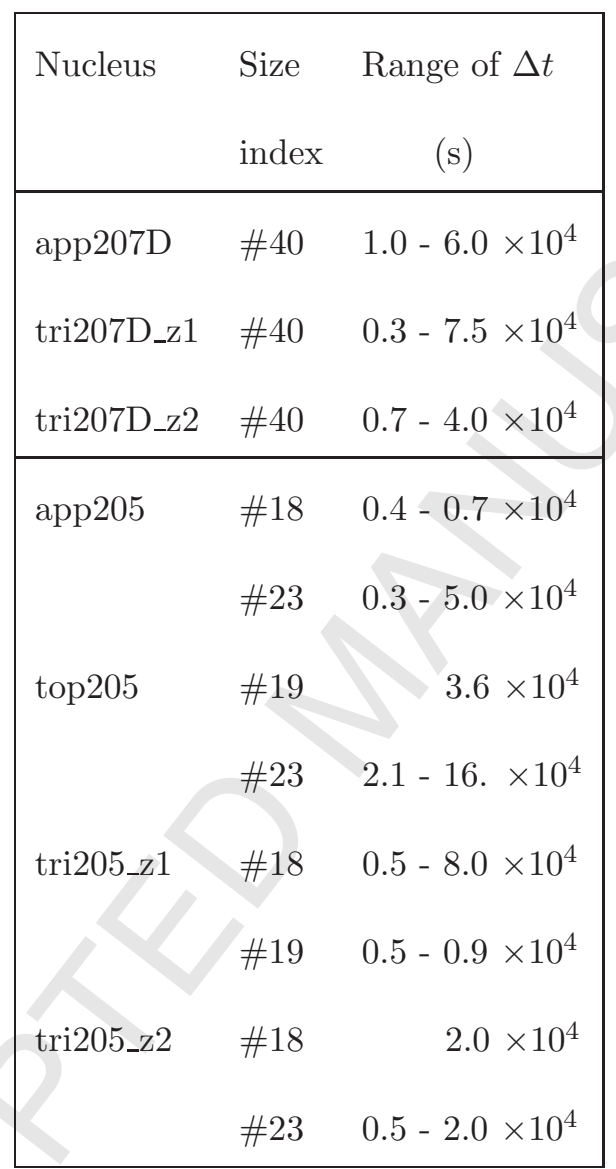

Table 4: Range of transit times $\Delta t$ between emission and re-impact found in the present study. 
considered processes; this is precisely why we omitted them from this study! These effects, will be considered in the following papers of our study. Until then, we consider that no adequate tool for reliably interpreting near-nucleus dust comae is at hand. As often occurs, the observations are in a sense in advance ahead of data analysis methods. This is in itself an important result, we believe, from our work.

Let us now summarize the present results. Most conclusions of paper D-I, where the nuclei were assumed spherical and homogeneous are confirmed by the present results where spherical-inhomogeneous and aspherical-homogeneous nuclei were considered. For instance, (1) re-impacting trajectories (forming volcanoes) are found again, and (2) the incorrectness of a-priori neglecting any one of the three forces considered when computing the nearnucleus dust coma is confirmed. This is a confirmation of the correctness of our method of "gradual sophistication" of the benchmark models: one sees clearly if and when new effects counter-act or not the previously considered ones. In addition to these earlier findings, the following new conclusions are brought forwards. (1) The areas of the nucleus with a reduced activity are susceptible to a volcanic-like bombardment by grains from more active areas. (2) The effect is more pronounced at small nucleus gas production, and might be particularly strong on the shadowed flanks of surface concavities which receive dust from other (sunlit) parts of the cavity; this cavity bombardment is associated with gas recondensation, and involves a large dust mass spectrum. (3) The non-sphericity of the gravitational field of aspherical nuclei plays an important role in the near-nucleus grain trajectories and grain number density. (4) The DMF method is confirmed to yield a highly accurate dust coma structure, as long as a suitable number of "dust fluids" are defined. (5) Even in the simple cases treated here, there is no evident way to decide whether a coma dust structure is due to nucleus asphericity or to nucleus inhomogeneity; and even the comparison between the gas and the dust structures does not allow to distinguish between these two processes.

\section{Acknowledgements}



07-01-00354.

\section{References} $549-551$ 353.

This work has received financial support from the French National Center for Space Research (CNES/CT) and from the Russian Foundation for Basic Research (RFBR) grant

Crifo, J.-F., 1988, Cometary dust sizing: comparison between optical and in-situ sampling techniques, Part. Part. Syst. Charact. 5, $38-46$.

Crifo, J.F., 1997, The correct evaluation of the sublimation rate of dusty ices under solar illumination, and its implications on the properties of P/Halley nucleus, Icarus, 130,

Crifo, J.-F., 2006, Physicochemical Simulation of the Near-Nucleus Coma: Present Achievements and Requested Future Developments Adv. Space Res. 38, 1923-1927.

Crifo J.-F., Itkin, A.L., Rodionov, A.V. 1995, The Near-Nucleus Coma Formed by Interacting Dusty Gas Jets Effusing from a Cometary Nucleus: I, Icarus 116, 77-112.

Crifo, J.F., Rodionov A.V., 1997a, The Dependence of the Circumnuclear Structure on the Properties of the Nucleus. I Comparison Between a Homogeneous and an Inhomogeneous Spherical Nucleus, with Application to Comet P/Wirtanen, Icarus , 127319 -

Crifo, J.-F., Rodionov, A.V., 1997b, The Dependence of the Circumnuclear Coma Structure on the Properties of the Nucleus, II. First Investigation of the Coma Surrounding an Homogeneous, Aspherical Nucleus, Icarus 129, 72 - 93.

Crifo J.-F., Rodionov A.V. 1999, Modelling the circumnuclear coma: objectives, methods and recent results, Planet. Space Sci. 47, $797-826$.

Crifo, J.-F., Loukianov ${ }^{3}$ G.A., Rodionov, A.V., Khanlarov G.O., Zakharov, V.V., 2002, Navier-Stokes and Direct Monte-Carlo Simulations of the Circumnuclear Coma: I Homo-

\footnotetext{
as Lukianov or as Lukyanov

${ }^{3}$ Unfortunately, due to fluctuations in the translation of Russian names, Loukianov is sometimes also written
} 
geneous, Spherical Source. Icarus 156, $249-268$.

Crifo, J.-F., Loukianov, G.A., Rodionov, A.V., Zakharov, V.V., 2003, Navier-Stokes and Direct Monte-Carlo Simulations of the Circumnuclear Coma: II Homogeneous, Aspherical Source. Icarus 163, $479-503$.

Crifo, J.F., Lukianov, G., Rodionov, A.V., Zakharov, V.V., 2005a, Direct Monte-Carlo and Multifluid Modelling of the Circumnuclear Dust Coma: I. Spherical Grain Dynamics Revisited. Icarus 176, 192-219.

Crifo, J.-F., Fulle, M., Kömle N.I., Szegő, K., 2005b, Nucleus-coma structural relationships, in: Comets II, M. Festou, H.U. Keller, H. Weaver (Eds), Tucson Arizona Press, pp. $471-503$.

Garmier, R., Barriot, J.P., 2001, Ellipsoidal Harmonic Expansion of the Gravity Potential: Theory and Applications, Celestial Mechanics and Dynamical Astronomy 79, $4,235-275$.

Keller, H.U., Knollenberg, J., Markiewicz, W.J., 1994, collimation of cometary dust jet filaments, Planet. Space Sci., 42(5), $367-382$.

Knollenberg, J., 1994, Modellrechnung zur Staubverteilung in der inneren Koma von Kometen unter spezieller Berücksichtigung der HMC-daten de GIOTTO mission, Ph. D. Thesis, Göttingen. in German

Hodges, R.R., 1994, Monte-Carlo simulation of the terrestrial hydrogen exosphere, J. Geophys. res. 99, A12, 23,229-23,247.

Lamy, P.L., Toth, I., Davidsson, B.J.R., Groussin, O., Gutiérrez, P., Jorda, L., Kaasalainen, M., Lowry, S.C., A portrait of the nucleus of comet 67P/Churyumov-Gerasimenko, 2007, Space Sci. Rev. 128, 23-66.

Lukyanov, G.A., Crifo, J.F., Zakharov, V.V., Rodionov, A.V., 2006, A new approach for modelling the dust dynamics in the near-nucleus coma, Adv. Space Res. 38, 1976-1982.

Mc Donnell, J.A.M., Lamy, P.L., Pankiewicz, G.S. 1991. Physical properties of cometary dust. in Comets in the Post-Halley Era, Ed. by R.L.Newburn \& J. Rahe, Kluwer Academic Press, Dordrecht, vol.2, 1043 - 1074. 
Rodionov, A.V., Crifo, J.F., Szegö, K., Lagerros, J., Fulle, M., 2002, An advanced physical model of cometary activity. Planet. Space Sci. 50, $983-1024$.

Whipple, F.S., 1982, Cometary nucleus and active regions, in Cometary Exploration,

Ed. T.I. Gombosi, Hungarian Academy of Sciences, Budapest, vol. 1, pp. 95 - 110.

Zakharov, V.V., Rodionov, A.V., Lukyanov, G.A., Crifo, J.-F., 2008, Navier-Stokes and Direct Monte-Carlo Simulations of the Circumnuclear Coma: III Inhomogeneous, Spherical Sources. Icarus 194, $327-346$. 